\title{
Ein Fall von Erweichung des Marklagers einer Großhirn- hemisphäre.
}

\author{
Von \\ Dr. Alired Fickler, \\ Oberarzt der Prov.-Irrenanstalt Obrawalde.
}

Mit 9 Textfiguren.

In dem Falle, ïber den ich im folgenden berichte, handelt es sich um eine Frau, bei welcher das Marklager der rechten Großhirnhemisphäre in ganzer Ausdehnung durch Erweichung zu Grunde ging; dies erfolgte in drei Schüben im 25., 30. und 32. Lebensjahr. Sie starb im 40. Lebensjahr. Bei der Sektion fand sich an Stelle des Marklagers der rechten Hemisphäre eine Cyste; die zentralen Ganglien waren zwar atrophisch, aber von der Erweichung selbst völlig verschont geblieben. Die Natur hatte also eine Veränderung gesetzt, wie sie so vollkommen wohl selten einem Experimentator geglückt ist: die Abtragung einer Großhirnhemisphäre unter Schonung der Zentralganglien; jede Verbindung zwischen Cortex und tieferen Hirnteilen war unterbrochen, nur in ihrem oralen Teil, vom frontalen Pol des Nucl. caudat. bis zum caudalen Pol des Nucl. amygd, standen die Zentralganglien an ihrer ventralen Seite mit der secundär degenerierten Rinde im Zusammenhang. Es bot sich so Gelegenheit, die klinischen und anatomischen Folgen einer Veränderung, die bei Tieren häufig experimentell herbeigeführt worden ist, auch beim Menschen zu untersuchen.

\section{Krankengeschichte.}

M. Z., Dienstmädchen; geboren am 17. Febr. 1864. Aufgen. am 15. Dez. 1893 wegen ,Epilepsie nach Schlaganfall".

A nam nese: Tochter zurzeit gesunder Eltern; der Vater soll früher ein Jahr lang „Krämpfe“" gehabt haben. Lernte in der Schule schlecht; körperlich entwickelte sie sich gut. War als Dienstmädchen in Ntellung; hat ein uneheliches Kind. Infektion geleugnet.

1889 im Mai merkte sie plötzlich bei der Arbeit eine Schwäche im linken Arm und linken Bein, Vorboten sollen nicht vorhergegangen sein; sie konnte zwar die Glieder noch gut bewegen, doch war die Kraft in ihnen gering. Noch mehr als die Schwäche aber waren ihr Gefühlsstörungen in den linkssedtigen Gliedern hinderlich: Hände und Füße waren plötzlich taub geworden, sie fühlte keinen Schmerz mehr, wenn sie sich stach oder stieß. Sie konnte mit der linken Hand nichts festhalten und verlor beim Gehen beständig den Pantoffel vom linken FuB. Ihren Dienst vermochte sic zunächst noch weiter zu versehen, in den folgenden Monaten aber wurde die Schwïche immer größer. In Herbst 1889 mußte sie ihren 
Dienst aufgeben, da sich zur Sichwäche und Gefühlslähmung noch Krampfa nf älle gesellten, die anfings tärlich zu mehreren Malen, später seltener auftraten. Im Beginn des Anfills merkte sie, dab dir fingarer der linken Hund sich zur Faust ballten, der linke Arm gebeugt wurde und der linke Mundwinkel sich zusammenzog, damn verlor sie dar Bewußtscin. In der linken Hand stellte sich allmählich cine Beugecontratur cin, die aber wieder zurückging, nachdem die $\mathrm{Kram} p \mathrm{f}$ anfälle seit Pfingsten 1893 wanz alusgesetyt hatten.

Aufnahmebofund am 15. Dez. 1893 .

GroBe, kräftig entwickelte, gut genährte frau. Schädel von mittlerer Größe, symmetrisch, ohne Narben. Stimrunzeln, Lidschluß gut. Augen parallel, frei beweglich. Pupillen gleich-und mittelweit; R/A, R/L I .

Untere zwo brittel des linken Facialis paretisch. T/ange ziemlieh gerade, ruhig vorgestreckt; Zäptehen weicht nach rechts ab.

An Zange, (atumen, Rachen keine Narben; Leukoderma am Nacken, oberer Brust und oberem Rückens.

Innere Organe ohme nachweisbare Veränderungen.

Die linksseitigen Ex tremitäten zeigen das gewönuliche Bild der Hemi. plegie: Arm in rechtwinkliger Beugestellung, die Hand proniert und in mäBiger Beugecontractur. Das Bein in Streckcontractur, die Spasmen kömen aber passiv mit geringer Kraft ïberwunden werden, Aktive Bewegung in allen Gelenken, in

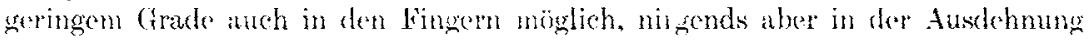
wic aut der rechten seite: Hebung des Armes bis zur Horizontalen. Beim Gehen wird das linke Boin sehleudernd, in einem halbkreisförmigen Bogen nach seitwärts, vorwärts bewegt. Hotorische Kraft in Arm und Bein beträchtlich herab. gesetzt. Beim Stehen ruht der Körper anf dem rechten Bein. 1)je Haut der linken Extremitäten, am stärksten distal, teigig, verdiekt und gerötet. Bein und Arm fühlen sich links bedeutend kühler an wie rechts, distal am kühlsten.

Keine Muskelatrophien.

Sensibilitä an der linken Körperseite bis zur Mittelliniestark gestört: Nadelstiche werden nicht schmerzhaft empfunden; Empfindung für. Kailte stark herabgesetzt, besser für Wärme erhalten. Muskelsimn ebenfalls stark becinträchtigt: die Kranke empfindet zwar, dak Arm und Bein bewegt wird, kamn aber nicht angeben, in welche Stellung sie gebracht sind. Berührungsempfindung a bogestumpft, Berïhrungen werden mangelhaft lokalisiert. Besser empfunden wirl rersehieden starker Druck. Unterschenkel links auf Druck schmerzhaft; dir. Sensibilitätsstörung ist am stäl'ksten ausgebildet in den distalen Abschnitten der Extremitäten.

Sehnenreflexe der linken Sieite stark erhoht.

Ful 3 klonus links.

1894. 23. Fobr. An Shatmlipen und After breite Kond ylume anfgetreten.

1. Juni. Kondylome geheilt. Beschäftigt sich mit Ntriimpfestopfen; ist ziemlich dement.

1. Nov. Seit vorgestern Krampfanfälle ohne Bewul3tscinsverlust: Kribbeln in der linken Körperhälfte, Zuckungen in den Muskeln der linken Körperseite, wolche in der Hand beginnen und auf Am und Gesicht fortschreiten; im linken Bein nur geringe Zackungen. Kann nicht sprechen, hört und versteht aber aller.

Heute 3 Krampfanfälle mit Bewubtseinsverlust und Zuckungen in der gosalmten Körpermuskulatur. Störung der Berührungs- und Schmerzemptindung

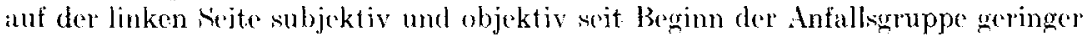
wir bei der Aufnahme.

4. Nor. Vorgestern 7 , gestern 6, heute 23 Anfälle von kurzer Dauer: Kopf und Augen werden nach links gedreht, Arme und Beine gestreckt, dann klonische

z. f. d. g. Neur. u. P'syoh. o. XY. 
Zuckungen in der Gesichtsmuskulatur, besonders links, in den Armen und Beinen, links stärker wie rechts, sowie starkc klonisch-tonische Krämpfe der Bulbi nach links.

In der anfallsfreien Zeit Sprache mangelhaft artikuliert, der eines kleinen Kindes ähnlich; verkennt Personen, hat Gehörstäuschungen.

6. Nov. Gestern 30, heute 124 Anfälle. Temperatur 38,0. Die Anfälle beschränken sich meist auf den Kopf (linker Mundwinkel nach oben gezogen, Kopf und Angen nach links gedreht; dann klonische Zuckungen in den linksseitigen Gesichtsmuskeln und horizontaler Nystagmus nach links); in linken Arm und Bein tonische Streckungen. Gefühl von Amcisenlaufen im linken Arm und Bein.

9. Nov. Vorgestern 50, gestern 28, heute 175 Anfälle. Ist immer nur kurze Zeit bei Bewußtsein und sehr unklar. Sprache sehwer verständlich, starke linksseitige Facialisparese, Brechneigung, Anfälle von wechselndem Aussehen: beginnen bald rechts, bald links, bald im Gesicht, bald in Arm oder Bein; dann verbreitet sich der Krampf meist über den ganzen Körper.

11. Nov. Gestern 176, heute 10 Anfälle. Ist sehr erschöpft, 'Temperatur 37,9 , Puls 96.

13. Nov. Anfälle haben seit gestern aufgehört. Erholt sich rasch. Ws zeigt sich, dab während des Status epilepticus eine linksseitige Hemianopsie auf beiden Augen eingetreten ist, die auf dem linken Auge von etwas größerer Ausdehnung ist wie auf dem rechten, sowie eine Blickläh mu ng des linken A uges; der Bulbus steht stark nasal gewandt und folgt weder nach außen, noch nach oben oder unten, ist in dieser Stellung völlig fixiert.

20. Nov. Facialisparese links geringer geworden. Sprache wieder deutlich. Die Beweglichkeit des linken Auges bis auf Abducensparese wieder gut. Hemianopsie bleibt bestehen. Sensibilitätsstörungen und Lähmung der linksseitigen Extremitäten, wie bei der Aufnahme.

1895. 23. April. Linksseitige bilaterale Hemianopsie unverändert; linkes Auge kann seit längerer Zeit wieder nach alken Richtungen gut bewegt werden. Gefühl links subjektiv besser geworden.

1896 Vom 26. März bis 27. April neuer Status epilepticus. Zahl der Anfälle voin 26. bis 31. März: 65, 28, 1, 42, 27, 35. Verlauf der Anfälle: Starke klonische Krämpfe in der linken Gesichtshälfte, Augen in konjugierter Deviation nach links; Nystagmus horizontalis nach links. Im linken Arm und Bein kurzc tonische Starre. Bewußtsein erhalten. In der a nfallsfreien Zeit Klagen über heftige Schmerzen in der linken Gesichtshälfte; Stirnkopfschmerz. Berührungsund Schmerzempfindung in der ganzen linken Körperhälfte abgeschwächt; Kopf und Spitze wurden aber ziemlich gut unterschieden.

1. bis 6. April. Zahl der Anfälle: 38, 35, 77, 42, 34, 55. Verlauf: Klonischer Krampf der linken Gesichtsmuskulatur und der Augenlidmuskeln, Bulbi starr nach links unten gerichtet, Kopf nach links gedreht. Im linken Arm und Bein beim Beginn des Anfalls Beuge- dann Streckkrampf. In der a nfallsfreien Zeit: Fast ununterbrochen Sopor; Kopf nach rechts gedreht; starke linksseitige Facialisparese; öfter Erbrechen.

7. bis 16. April. Zahl der Anfälle: 43, 41, 54, 35, 25, 28, 35, 29, 30, 38; Verlauf der Anfälle ähnlich wie vorher. In der a nfallsfreien Zeit: Sopor geringer geworden, Bulbi stehen stark nach rechts, können aktiv nach oben und unten nur wenig, nach links zu gar nicht bewegt werden. Dazu Ptosis, die am 10. April vollkommen ist, so daß die Augen gar nicht geöffnet werden können, aber an den folgenden Tagen links stärker ist wie rechts. Sprache verwaschen, mangelhaft artikuliert. Jinksseitige Extremitäten völlig gelähmt. Facialisparese, Kopfstellung wie vorher. Starke Schmerzen in der linken Cesichtshälfte und linkem FuB. 
17. bis 27. April. Zalhl der Anfälle: 25, 25, 23, 18, 27, 15, 17, 10, 13, 9, 5. Anfälle leichter und kürzer wie vorher, bestehen in klonischen Zuckungen der linken Mundmuskulatur und der distalen 'Teile der Extremitäten; am Schluß des Status beschränken sie sich auf die linke Hand. Lähmungen am Finde des Status wie auf seiner Höhe.

15. Mai. Augen und lider aktiv wieder gut beweglich, Sprache deutlich.

17. Juni. Die lähmung des Facialis und der Extremitäten ist allmählich bis auf den früheren Grad wieder zurückgegangen; die Hemianopsie dauemd unverändert. Sensibilitätsstörung nicht mehr so hochgradig, wie bei der Aufnahme.

1897. März bis April. Hypuchondrische Wahnideen. Habe kein Herz mehr, könne niemals gesund werden usw.

1898. 16. bis 21. Sept. Täglich mehrere leichte, epileptiforme Anfälle ohne Bewußtseinsverlust, bestehend in Zuckungen in Gesicht und teilweise auch in den linksseitigen Extremitäten. Die Anfälle können hervorgerufen werden, wenn die Kranke die Augen stirk nach links wendet.

In den folgenden Jahren bis zum Tode traten keine wesentlichen weiteren Veränderungen auf, abgesehen von der Demenz, die nach dem letaten Status "pileptieus noch größer geworden und mit paranoischen Ziigen verknüpft war, war das Krankheitsbild dauernd das folgende:

A ugenbewogungen nach allen Richtungen möglich; Bulbi parallel. Pupille $\mathrm{n}$ reagieren auf Jicht und Akkomodation, die linke ist etwas enger wie die rechte. Bilaterale linksseitige Hemianopsice.

Gehör beiderseits gut. Untere zwei Drittel des linken Facialis paretisch. Zunge weicht beim Ausstrecken otwas nach links ab. Gaumensegel steht links atwas tiefer und wird weniger gut gehoben wie rechts.

Sprache otwas stockend und explosiv, sonst aber nicht gestört.

Motilität: Steht und geht wie ein Hemiplegiker; kann Treppen steigen. Beschäftigt sich mit strümpfestopfen, wobei sir den Strumpt zwischen linken Arm und Thorax klemmt. Linker Arm und linke Hand in Beugecontratetur, Oberarm an den Thorax adduziert. Aktive Streckung im Ellenbogengelenk gering, besser Abduktion und Helnung des Oberames (fast bis zur Horizontalen). Finger aktiv fast gar nicht beweglich, in Beagecontracturstellung. Linkes Bein in Streckontractur, aktive Bewegungen im Hüf- und Kniegelenk in beschränktem Maße möglich; Fuß steht in mäßiger Spitzfußstellung und ist cinwärts rotiert; Zehen in den Grundphalangen dorsil flektiert. Fuß und Zehen können aktiv nul sehr wenig bewegt werden. Muskeltonus links erhöht. Muskulatar am linken Arm und Unterschenkel etwas atrophisch.

Sensibilität auf der linken Hälfte des Körpers in allen Qualitäten mehr oder weniger gestört, am Rumpf, Oberarm und Oberschenkel in geringerem Grade wio an den distalen Teilen der Extremitäten. Berührungsempfindung ïberall abgestumpft, an Hand und Fuß fast aufgehoben. Nadelspitze und Knopf wird ziemlich gut untersehieden, nur an Hand, Unterschenkel und Fuß ist die Unterscheidung unsicher. Für Nadelstiche besteht am Rumpf, Oberarm und Oberschenkel Hyperalgesie, an Hand und Fuls werden erst tiefere Nodelstiche schmerzhaft empfunden. Kalt und Warm wird bei geringer Differenz mangelhaft untersehieden, am Rumpf rufen höhere Wärme- und Kältegrade Schmerzäuberungen hervor.

Lokalisationsvermögen schlecht an den distalen Terilen der Extremitäten, sonst links ziemlich gut.

Lagesimn üborall stark herabgesetzt; sie fühlt, daB Arm und Bein bewegt werden, kann alber Richtung und Stellung nicht angeben.

Die Seh nenrefle xe sind links sämtlich erheblich gestejgert, die Hautreflexe nicht anslöshar. 
Während des Jahres 1903 verfiel Patientin geistig und körperlich immer mehr. Ende Nov. 1903 entwickelte sich im Anschluß an einen Fall ein subcutaner Absceß in der Gegend der unteren Brustwirbelsäule; unter meningitischen fymptomen starb sie: am 21. Jan, 1904.

\section{Leichenbefund:}

Eiterige Meningitis. Totale Erweichung des Marklagers der rechten Großhirnhemisphäre; sekundäre Degeneration der Zentralganglien, der absteigenden Bahnen und der linken Kleinhirnhemisphäre.

Chronischer Magen-Darmkatarrh.

Atrophie der Lunge, Leber, Milz. Braune Atrophie des Herzens.

Lungenödem.

Makroskopischer Befund der Sichädelhöhle:

Schädeldach rechts verdickt, desgleichen die Dura. Innenfläche der Dura glatt und glänzend.

Pia besonders an der Konvexität des Großhirns trüb und ödematös, über den Furchen gelblich infiltricrt; über der rechten Hemisphäre außerdem diffus verdiekt.

Rechte GroBhirnhemisphäre eingesunken. The Windungen erscheinen plattgedrückt. Beim Betasten schwappt sie wie ein nicht völlig mit Wasser gefüllter Sack. Beim Einschneiden entleert sich aus ihr in großer Menge eine trübe, braun-rötliche Flüssigkeit; es zeigt sich, daß das ganze Marklager der Hemisphäre geschwunden ist; sie besteht nur noch aus der stark verschmälerten, fest mit der Pia verwachsenen Rindenschicht und in der Gegend der Zentralganglien aus einer bis zu $2 \mathrm{~cm}$ dicken Gewebsmasse, die derb und graugelblich ist und eine Zeichnung nicht mehr deutlich erkennen läßt. Die Oberfläche dieser Gewebsmasse ist zottig, während die Rinde an ihrer dem cystischen Hohlraum zugekehrten Fläche ziemlich glatt ist; die Innenwand der Cyste ist der Form nach das Negativ der Großhirnoberfläche: entsprechend den Sulci ragen überall Septen in den cystischen Hohlraum vor. An der Konvexität ist die Rinde stärker geschrumpft, als an der Konkavität, ihre Breite beträgt dort etwa 1, hier fast $2 \mathrm{~mm}$.

Rechter Hirnstiel, rechte Hälfte der Brïcke und Medulla oblongata stark verkleinert.

Linke Hemisphäre des Kleinhirns in allen ihren Bestandteilen fast auf die Hälfte des Volumens der rechten reduziert.

Rinde, Mark und zentrale Ganglien der linken Großhirnhemisphäre zeigen keine pathologischen Veränderungen, nur ist ihr Volumen beträchtlich kleiner wie das einer normalen Hemisphäre. Die Gehirnsubstanz ist blutreich und feucht. Der Balken ist völlig geschwunden, ebenso der rechte Fornix; der linke Fornix ist stark atrophisch.

Die Gefäße der Basis haben normalen Verlauf; die der rechten Hemisphäre zugehörigen Gefäße sind um mehr als die Hälfte dünner, wie die der linken; ihre Wandungen sind gleichmäßig verdickt; nirgends findet sich ein Verschluß des Gefäßlumens.

Zur Orientierung über die Ausdehnung der Zerstörung füge ich hier die $\mathrm{Ab}$. bilding cines Frontalschnits durch das Großhirn bei.

\section{Mikroskopischer Befund:}

Zur mikroskopischen Untersuchung wurde das Großhirn in 10 proz. Formollösung gelegt; das Rürkenmark war leider versäumt worden herauszunehmen. Von der Hirnrinde beider Hemisphären wurden Schnitte aus den verschiedenen Lappen mit Hämatoxylin-Eosin, nach Weigert-Pal, van Gieson, Nissl und 
Bielschowski gefärbt. Die zentralen Ganglien der rechten seite warden bis auf einen schmalen streifen durch das oralste Thalamusgebiet, welches nach Nissl gefärbt wurde, in frontale Serienschnitte zerlegt und diese abwechselnd nach Wejgert-Pal, von Gieson und mit Camin gefärbt. In gleicher Weise wurden Sehnitte aits allen Gegenden der Zentralganglien der linken Hemisphäre, Vierhïgelgegent. Brücke und Medulla oblongata behandelt. Von versehirdenen

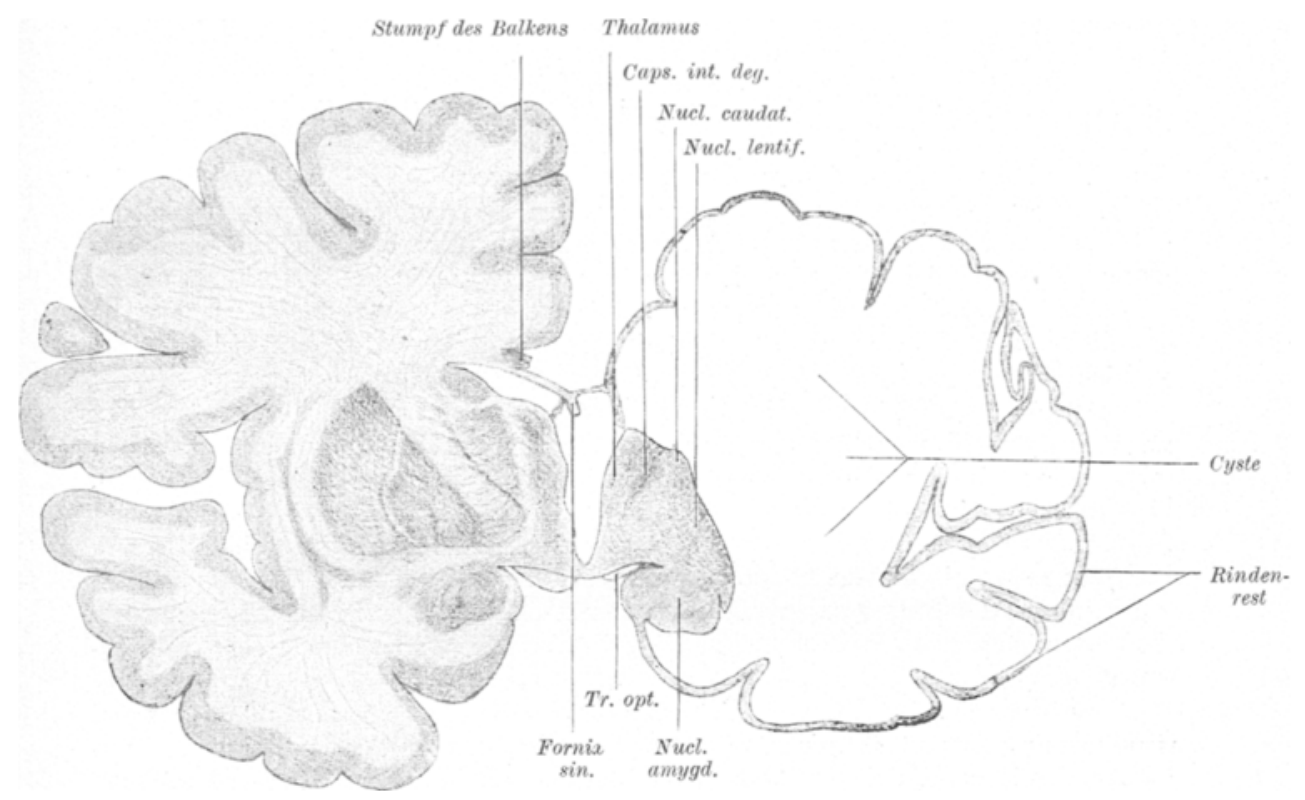

Fig. 1. Frontalschnitt durch das Großhiru ca. $1 / 2 \mathrm{~cm}$ hinter dem Chiasmal.

Gegenden der Brücke und des Kleinhirns wurden Nissl-Präparate angefertigt. Vom Kleinhirn wurden außerdem Weigert-Pal-, Carmin-und Biclschowski. Iräparato heroestellt.

\section{Rechte Hemisphïre.}

Die groben GefäBe in der Pia (Art. ccrebr. ant., Foss. Sylv. und cerebr. post.) sind stark kontrahiert; Intima und Media sind in mäßigem Grade gleichmäßig verdickt. Nirgends finden sich cireumseripte Verdickungen, Infiltrationen, Aneurysmen oder Gummata in der Gefïßwand.

Die Hirnrinde ist in allen Gegenden reduziert, aber nicht überall gleich stark. Am relativ besten ist sie jeweils in den Furchen erhalten, auf der Höhe der Windungen ist sie am stärksten geschädigt. Territorial betrifft die stärkste Dr. gencration die mittleren zwei Drittel der Konvexität und medialen Seite, etwas besser ist exhalten der frontale und oceipitale Pol, am besten die basalon Rindenteile; die beste erhaltene stelle ist der kandale Teil des Gyrus rectus.

Von den Ganglienzellensehirhten fehlen auch an den am besten erhaltenen Stellen die großen Pyramidenzellen der 3. Schicht; die Zellen der polymorphen und Körnerschicht sind dichter aneinander gelagert, sklerotisch and rarefiziert; besonders ist die Zahl größurerZellen in der polymorphen Schi ht sehrgering, die Riesenpyramiden der Zentralwindungen fehlen durchaus auch an den besset erhaltenen 
Stellen dieser. Windungen. Auch die Zellen der 2. S'chicht liegen dichter ancinander, ihre Zahl aber scheint nicht vermindert, ebensowenig wio die der l. Schicht.

An den weniger gut erhaltenen Stellen sind auch die Körner- und polymorphe Zellschicht hochgradig rarefiziert; die 1. und 2. Sehicht ist ebenfalls ärmer an Zellen.

An den stärkst geschädigten Stellen ist von Zelschichten nichts mehr wahrzunehmen, hier und da sieht man noeh eine ganz verkümmerte Ganglienzelle, die, wie die Verfolgung des Präparates ergibt, der Schicht der kleinen Pyramidenzellen angehört. Die Rinde ist völlig in ein gliöses Gewebe mit wenig Zellen umgewandelt. das ziemlich zahlrejche Capillaren aufweist. Die Glia ist auch in den besser erhaltenen Stellen stark gewuchert und hier reich an Zellen.

Von Nervenfasern sind an den besser erhaltenen Stellen die Fibrae propriae in größerer oder geringerer Anzahl vorhanden; die Radiärfasern sind an diesen Stellen, wenn auch stark rarefizjert, noch erkennbar, auch in der äußeren 13aillargersehen Schicht finden sich noch Nervenfasern; die Zahl der übrigen tangentialen Nervenfasern ist sehr gering. Die molekulare Substanz ist hochgradig geschrumpft in der 4. und 5. Schicht, in geringerem Grade auch in den äuBeren ichichten.

An den stärkst geschädigten Rindenstellen sind auf Wejgert-Präparaten Nervenfasern überhaupt nicht mehr,zu finden; in Biclschowski-Präparaten sieht man noch hie und da eine Faser in schräger oder tangentialer Richtung ziehen.

Das Hemisphärenmark ist restlos zugrunde gegangen, mit ihm auch das ('laustrum. Die Wand der an seine Stelle getretenen Höhle wird gebildet durch die Hirnrinde, die zentralen Ganglien und dio Tola chorioidea; dor Seitenventrikel ist in der Höhle aufgegangen. Die nervösen Teile dieser Wand zeigen an der der Höhle zugekehrten Scite mehr weniger starke (\$liawucherung und Ependymverdickung.

Mit dem Hemisphärenmark ist auch der Balken völlig zugrunde gegangen. Als letzter Rest von ihm fíndet sich unter dem (iyrus fornicatus der linken Hemisphäre ein in der Hauptsache aus Gliagewebe bestehender Stumpf (Fig. 2, 3); von ('ommissurenfasern ist in ihm nichts mehr vorhanden. Dagegen findet sieh an seiner dorsalen Oberfläche ein (ianglienzellenhaufen, die linke Stria Lancisii, erhalten. Der Haufen wird durchflochten von feinen Nervenfasern; zu ihm treten Fasern vom Cingulum der linken Hemisphäre und zichen Fasern in vertikaler Richtung durch den Balken hindurch zu Septum pellue. und Fornix, vereinzelt auch zum Stratum subendymale der linken Hemisphäre (Fibrae perforantes corp. eallos.). Vom ('ingulum aus gelangen auch direkt, an der Stria Lancisii lateral vorbeizichend, Fasern an die ventrale Fläche des Balkens.

Vom Septum pellucidum ist nur die linke Hälfte erhalten. Seine Fasern zichen teils von der unteren Fläche des Balkenstumpfes zum Stratum subendymale, teils durchziehen sie den Balken in schräger Richtung nach auBen oben, gelangen in die Eintrittszone des Balkens in die Hemisphäre und scheinen dort zum ('ingulum umzubiegen.

Der rechte Fornix ist ebenfalls völlig zugrunde gegangen; der linke ist auf fast ein Drittel seines Volums reduziert. Die hintere Hälfte mit der Lyra war leider bei der Präparation verloren gegangen.

Um zunächst die nit dem zentralen Richapparat in Verbindung gebrachten Hirnteile vorwegzunehmen, so zeigt die Rinde des Tuberculum olfactorium ähnliche Veränderungen wie die der besterhaltenen Teile der Hirnrinde; die Subst. molec. ist in den oberflächlichen wie in den tieferen Schichten, in diesen stärker, reduziert. Die Ganglienzellen der oberflächlichen beiden Schichten haben wenig gelitten; doch finden sich auch in ihnen, wie in den Riechinseln sklerotische Zellen. 
Stärker reduziert sind die Schicht der großen Pyramiden und der polymorphen Zellen; vereinzelt sind aber hier große Pyramidenzellen erhalten. Die Nervenfasern sind stark reduziert (Fig. 2); die meisten von ihnen zieben zur Commissura ant., wenige medial-, noch weniger lateralwärts.

Die Commissura ant. hat eine erhebliche Einbuße an Fasern erlitten; ihre Fasern ziehen zu den basalen Stirnwindungen und zum Tuberc, olf.; von Sichläfenanteil ist fast nichts erhalten geblieben.

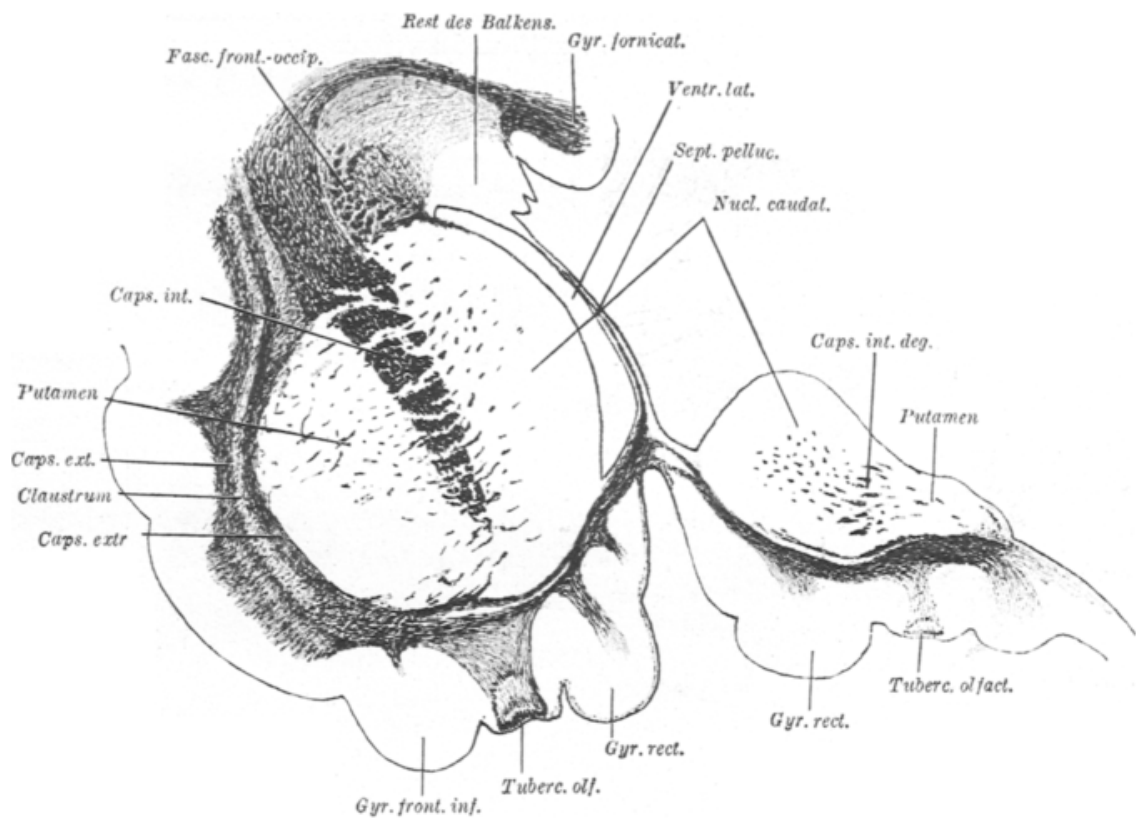

Fig. 2. Frontalschnitt durch die zentralen Ganglien in der Gegend des Thberculum olfactorium. Wiigert-Pal. Vergr. $1: 2$.

Gut erhalten ist die Taenia thalami (Fig. 4, 5) und das Ganglion haben ulac (Fig. 6). Die Taenia erhält Zuzug von Fasern in den caudalen zwei Dritteln des Thalamus vom Linsenkern und den ventralen Thalamuskernen her (Fig. 5, 6); jener verläuft im Strat. zonale. Vom Ganglion habenulae aus zieht der Fasei. culus retroflexus in unverminderter Stärke zum Hirnstiel.

Das A m monshorn ist im ganzen stark degeneriert (Fig. 6), der Hippocampusanteil weniger wie der der Faseia dentata. Vom Hippocampusanteil ist der ventrale Teil besser erhalten wie der dorsale. Der ventrale verhält sich ähnlich wie die besser erhaltenen Rindenstellen sonst (Molekularsubstanz in den tieferen Sichichten geschrumpft, Fehlen der großen Pyramidenzellen, Atrophie und Sklerose der polymorphen Zellen); die Ganglienzellen des Subiculums sind nur einfach atrophisch. Das dorsale Blatt des Hippocampusteils dagegen ist stark degenericrt; hier finden sich nur vercinzelt geschrumpfte Ganglienzellen, nur an der medialen Umbiegungsstelle zur Fase. dent. sind sie etwas zahlreicher in der Pyramiden. schicht erhalten. Die beiden Blätter des Stratum zonale des Hippocampusanteils sind andeutungsweise erhalten, bestehen aber aus sehr wenig Fasern; der Alveus 
fehlt vollkommen. Sekn stark degeneriert ist die Fascia dentata; das Stratum granulosum ist vollkommen gewehwunden; die polymorphe Zellschicht enthält nur vereinzelte stark atrophische (ianglienzellen. Aus dieser Zellschicht zichen vereinzelt Fasern, zu denen sich einige aus dem dorsalen Blatt des Hippocampusantcils gesellen, in der Fimbria dorsalwärts. Im Strat. radiat. der Fascia dent. finde’n sich keine Nervenfasern.

Mit dem Hemisphärenmark sind auch völlig degeneriert alle von der Hirnrincle in der Caps. int. zu den tieferen Hirnteilen ziehenden Fasern (Fig. 2- 6). Dagegen durchziehen überall in wechselnder Menge Fasern die innere Kapsel, die einmal den N. caudat. mit dem N. Ientif. und zweitens den Streifenhügel (be-

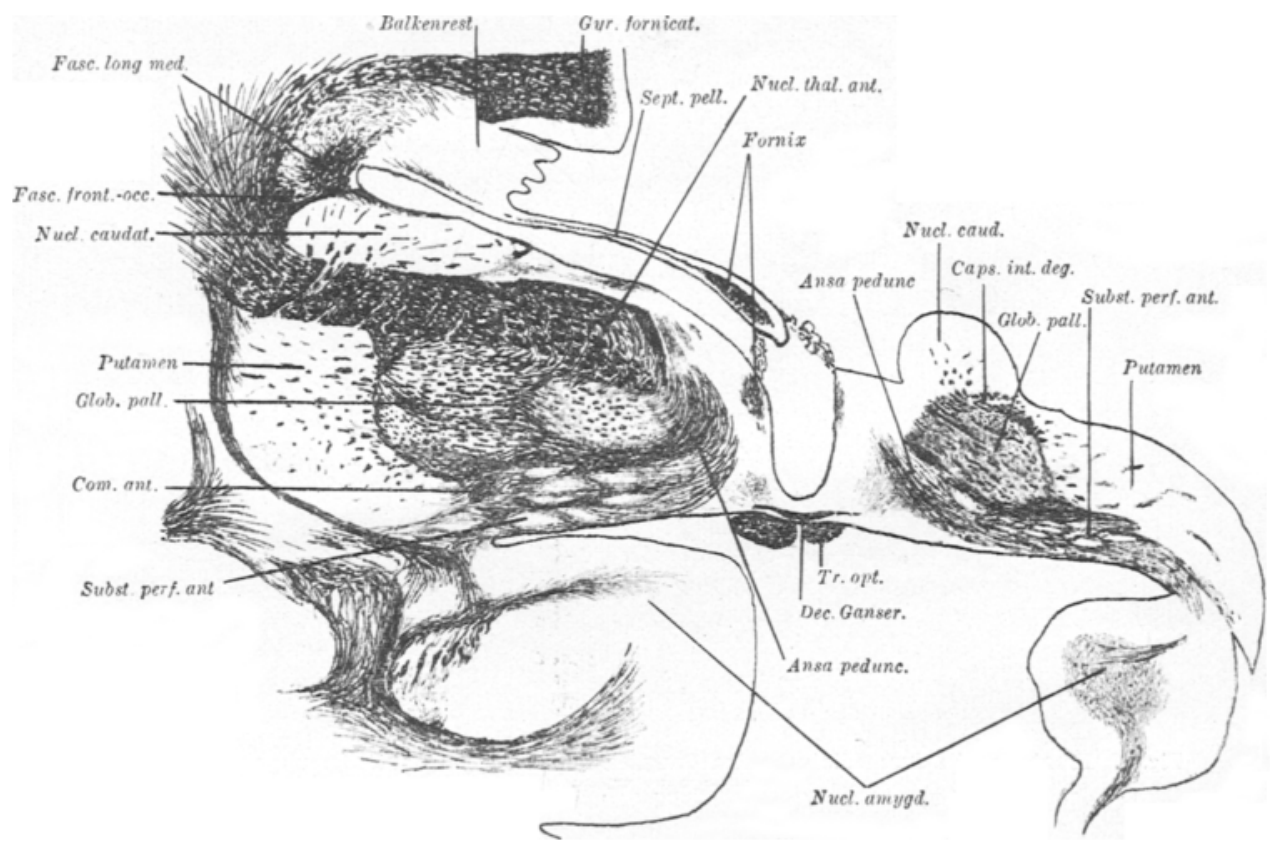

Fig. 3. Frontalsehnitt durels die zentralen Ganglien dicht hinter dem Chiasma. Weigert-pal. Vergr. $1: 2$.

sonders den Linsenkern) mit dem Thalamus verbinden (fronto-rlorsaler 'Tril des Fase. striothalamicus). Im vorderen Schenkel der inmeren Kapsel sind die Fasern zwischen Streifenhüget und Thalamus zu dickeren Bündeln geordnet (Fig. 2, :3), verlaufen in ventro-medialer Richtung und splittern sich besonders im medialen und vorderen sichhïgelkern auf; in dem mittleren und hinteren Teil der inneren Kapsel verlaufen diese Fissern mehr verstreut und untermischen sich mit de'm Rest der Lan. med. ext., so daßs sich ihr Fude nicht bestimmen läßt; sie kommon hauptsächlich aus dem (ilob. pallid. Mit den Fasern zwischen Nucl. candat. und Nucl. lentif. durchzieht auch der Faserzug vom Linsenkern zur Taenia thal. die innere Kapsel (Fig. 4, 5); dicser laserzug tritt in enge Berührung mit der Stria term., scheint mir aber keine Fasern an sie abzugeben. Wehr dentlich tritt danm ferner in den hinteren Abschnitten der inneren Kapiel die Linsenkerusehlingr hervor (Fig. 4, 5); sie erseheint hier röllig isolient und erinnert all das Bild, walehes 


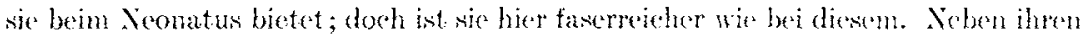
Ausstrahlungen in den 'Thalanus, das 'Tub. eince. und 'orp. subthal. rendet sije anch Fasern in die Subst. nigr.

Der Nucleus caudatus ist im Schwanzteil in den frontalen Ebenen des vorderen ('orp. mammil. an einer Stelle in die Erweichung des Markes miteinberogen; dic Stelle hat etwa die (iröße eines Gerstenkorns; um die erwejehte Stelle herum ist das (iewebe dess $\mathrm{X}$. caud. stark infiltriert, die (ianglienzeilen und Nervenfasem sind in der nächsten Naxhbarschaft ganz geschwunden und auch in der weiteren. allmählich an Intensität abnehmend, rarefiziert. Im übrigen ist dic Form des N. caud. völlig erhalten, nur seine Größe ist reduziert und zwar im Kopfteil auf fast die Hälfter, im Schwanzteil um ein Viertel seines Volums. Daß3 an der Sehrumpfung die kleine erweichte Stelle im Schwanzteil die Schuld trage, ist nicht anzunehmen; stehen doch die rinzelnen Teile des $\mathrm{N}$. cand. untereinander nicht durch längere Assoziationsbahnem in Verbindung. Sie ist jedenfalls die Folge des Ausfills der Verbindung mit dem ('ortex: das Hemisphärenbïndel und damit die von ihm in den $X$. caud. einstrahienden Xervenfasern sind völlig zugrunde gegangen; der dorsolaterale 'leil des Kernes ist daher ganz frei von Nervenfasern. Auch die Zahl der (ianglienzellen ist in diesem Teil geringer, während sie sonst nicht wesentlich herabgesetzt ist; doch finden sich vereinzelt sklerotische Zellen. Stärker reduziert ist die Subst. molec., besonders im Kopfteil, und die Norvenfitserbündil wibd vorschmälert (Fig. 2, 3).

Ahnileh ist das Verhalten des Xuel. Ientiformis. In der gleichen Gicgend wie beim X. caud. hat die Frweichung an einigen kleineren Stellen iluf das Putane'n übergegriffen und zu denselben Veränderungen geführt wie dort. Sonst ist anch der linsenkern der Form makh völig erhalten. Siein Volum ist um cotwa cin Viertel reduzient.

Im Putamen fehlen wieder die vom $\mathrm{Hemisphärenmark} \mathrm{einstrahlenden} \mathrm{liaxer-}$ bïndes vëllig, und die feinsten Nerventasern und die Molekularsubstaim sind reduziert; auch die radiären Fascrbündel sind :n Zahl vermindert und diunner. Die Ganglienzellen sind in den lateralen Partien spärlich. besonders fohlen die größeren Zollen; ein 'Leil der erhaltenen ist sklerotiseh.

In Glob. pallid. ist dic molekulare Substanz ebenfalls reduziert, dic Fasolbändel sind verschmälert, in cler Lamina med. sind besonders dic vertikal verlaufenden Fasern geringer an Zahl. Die Ganglienzellen sind ziemlich gut rohalteis: nur die kleineren seheinen weniger zahlreich zu sein wie auf der linken keite.

In der lateralen Peripherie des Putamen finden sich öfter alte Blutalustritte. IIII Gefäße herum (Hämosiderin teils in Zellen, teils frei im (ieweb).

Der Nucl. a mygdalae (Fig. 3, 4) hat nur wenig gelitten. Feine (amglienzellen sind gut erhalten; die Molekularsubstanz ist nur wenig reduziert. Von der angren\%enden Rinde her treten Nervenfasern in ihn rin; aus srinem oralen Tril zichen Fasem in die Gegend des gut erhaltenen X. ansas pedune, und scheinen mit dem unteren Thalamustiel, der an fisern roduziert ist, zum Thalamus zu gelangen. I te mittleren Drittel des Linsenkerns treten zu desisein basaler Mark-

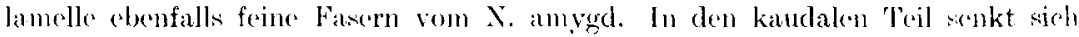
die Striat terminalis ein; sie ist äberall gut erhalten und hebt sich infolge des Wogfalls der ungebenden Xervenfasern scharf ab (Fig. 4, 5. 6); sice endet in den medialen und zentralen Partien des X. amygd.

An stärksten gesehäligt von allen Zontralganglien ist der Thalam ms; ab-

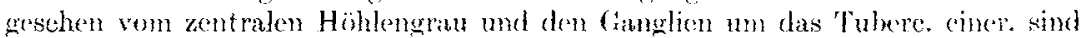
alle Kerne mehr oder woniger stark in Mitleidenschaft gezogent.

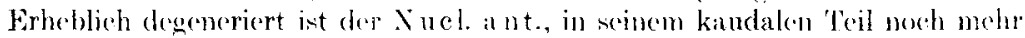
wie im oralen. Von der Markkapesel sind nur geringe Andeutungen in Gestalt rer- 
einzelter Fasern erhalten. Lir crhält geringen Faserzuzug vom Linsenkern her durch die innere Kapsel (siehe oben) und durch das Vieq d'Azyrsehe Bündel, das aber auch nur aus wenig Fasern besteht (Fig. 4). Seine Ganglienzellen sind größtenteils zugrunde gegangen, die resticrenden sind meist sklerotisch; din Subst. molec. ist stark geschrumpft.

Am stärksten degeneriert unter den Thalamuskernen ist der $\mathrm{Nucl}$. lat. in allen seinen Teilen. Er ist bis auf etwa den 6. Teil seines Volumens zusammengeschrumpft. In den oralen zwei Dritteln wird er gebildet durch ein weitmaschiges (ilianetz, in dessen Masehen nur ganz vereinzelt, an zahlreichsten noch dorsal und ventral, feine Nervenfasern sich finden. Von Ganglienzellen ist nur ganz vercinzelt mal eine erhalten, am häufigsten im ventralen Teile und in der Nähe der

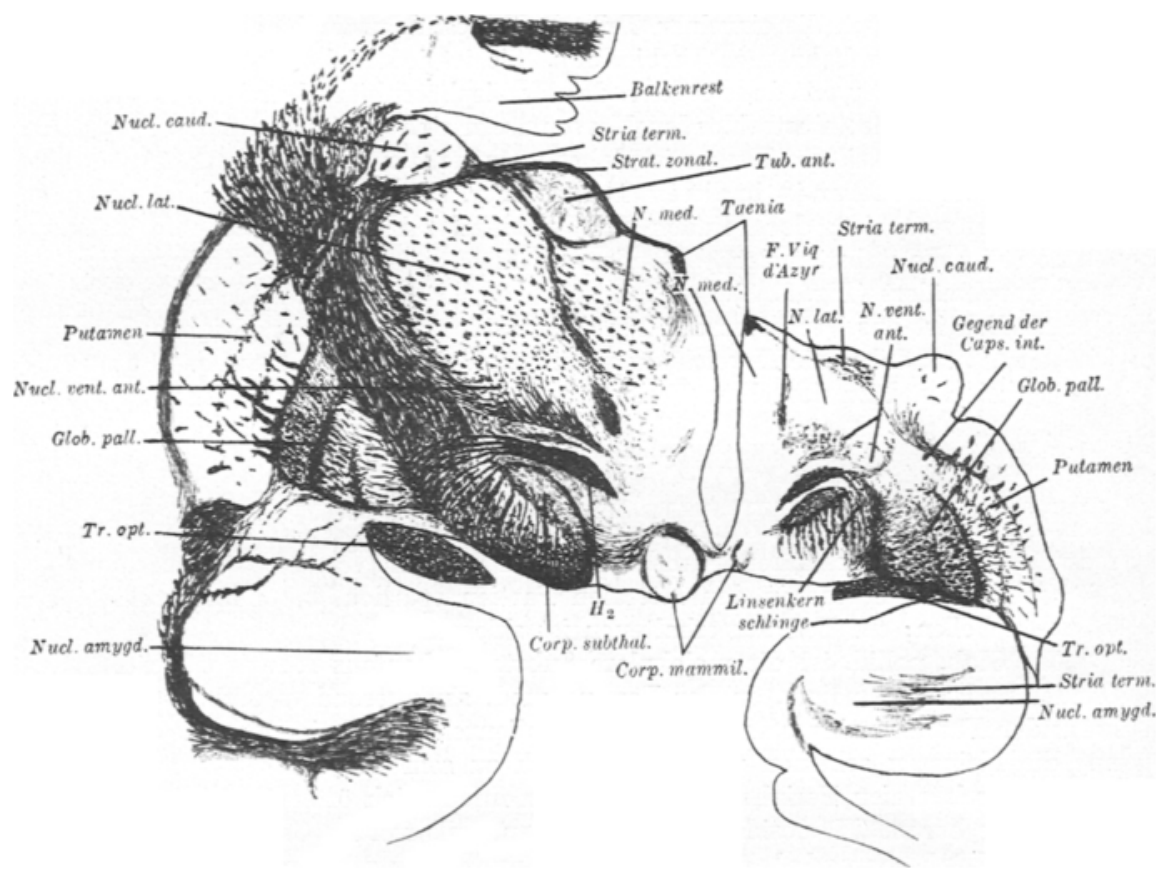

Fig. 4. Frontalschnitt durch die zentralen canglien am oralen Fude des Corp. subthal. Wrigert-Pal. Vergr. $1: 2$.

Lam. med. ext., hier sieht man ab und zu auch noch eine der großen Zellen, die sich hier finden. Das Gewebe ist stark geschrumpft, die perivasculären Lymphräume infolgedessen stark erweitert, so daß es den Findruck macht, als sei der Kern siebartig durchlöchert. Stellenweise finden sich Reste alter Blutaustritte um sonst normale GefäBc. Das kaudale Drittel des N. lat. besteht aus einem dichteren Glianetz, die Erweiterung der perivasculären Lymphräume ist aber auch hier vorhanden. Der Untergang der nervösen Substanz ist hier fast ebenso stark wie in den oralen Dritteln, nur im ventralen Teil finden sich von der Lam. med. ext. ausstrahlend etwas mehr feine Nervenfasern und auch in etwas größerer Zahl erhaltene Ganglienzellen.

Sehr stark degeneriert in scinem oralen 'Teil ist auch der $\mathrm{Nucl}$ med. Die dorsale Hälfte weist gar keine Ganglienzellen und Nervenfasern auf; in der ven- 
tralen finden sich einige Bündel zarter Nervenfasern, die kaudalwärts an Zahl zunehmen, und auch einfach atrophische größere und kleinere Ganglienzellen sind in einiger Anzahl vorhanden. Besser ist der kaudale Teil des Nucl, med. Hier weist auch der dorsale Teil (med. a) feine Faserbündel und zum Teil atrophische (ianglienzellen in etwas größerer Anzahl auf; der ventrale Teil (med. b und e) ist crfült von eine'm dichten Geflecht von Nervenfasern, zwischen denen zahlreiche ( ianglicnzellen, auch größ3ere von meist guter Struktur erhalten sind; die Subst. molec. ist übcrall stark reduziert.

Vou den ventralen Kerngruppen haben am stärksten golitten der hintere Kern, der fast völlig geschwunden ist, und der $\mathrm{N}$. vent. ant., dann vent. a und $\mathrm{c}$, am relativ besten ist vent. b erhalten. In allen Kerne finden wir Einstrahlungen von

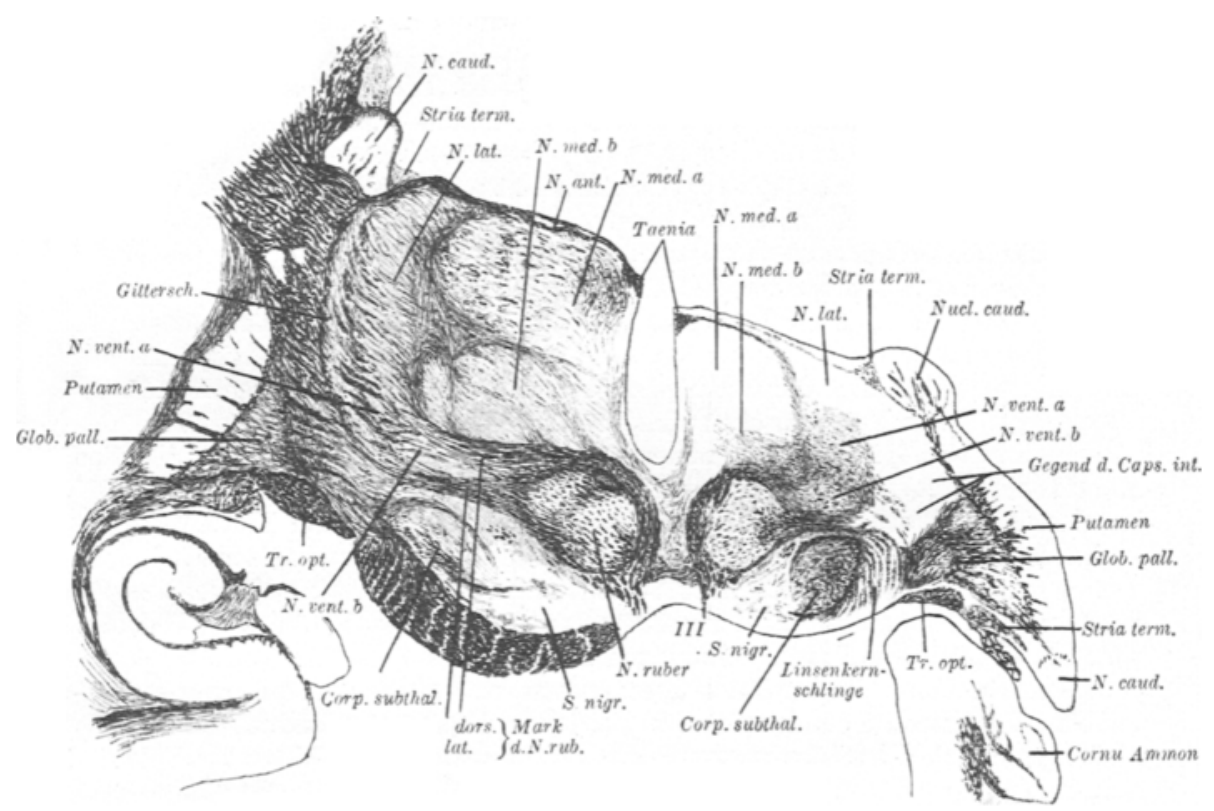

Fig. 5. Frontaischnitt durch die zentralen (ianglien, Nite des Nucleus ruber. Weigert-Pal. Vergr. 1 : :2.

Nervenfasern von der oberen Schleife, Lam. med. ext. usw. her, am dichtesten ist das (ieflecht, welches von diesen Nervenfasern gobildet wird, im N. vent. c. Die: molekulare Substanz ist überall reduziert. Die Zahl der Ganglienzellen ist im $\therefore$ vent. ant. beträchtlich verringert, (ein großor Teil zeigt sklerotische Veränderungen; im $N$. vent. a und e sind sie zahlreicher vorhanden und meist normal oder einfach atrophisch; ein kleiner Teil ist stärker geschrumpft. Im N. vent. b ist die Zahl der Ganglienzellen zwar auch noch erheblich geringer wie links, doch sind dic vorhandenen meist gut erhalten.

Der $x$. arejformis ist um etwa ein Drittel in seinem Areal reduziert. Die Nervenfasern sind spärlicher wie auf der Jinken Seite; dic Ganglienzellen an Zahl wenig vermindert, aber atrophisch bei gut erhaltener Struktur.

Hochgradig degeneriert ist wieder das Pulvinar. Sein Areal ist ähnlich zusammengeschrumpft wie das des Nucl. lat. Die allermeisten Ganglienzellen sind zugrunde gegang(ll, vereinzelte kleine sind crhalten. Ganz vereinzelt finden 
wich feine Nervenfasern, die von der lateralen Seite cinstrahlen; ein wenig zahlreicher sind sic im kaudalen 'Teil, wo vom ('orp. genic. ext. her Züge durch die Lam. med. ext. zum Pulvinar treten. Hier findet man auch etwas häufiger eine kleine Ganglienzelle. Im übrigen besteht das Pulvinar aus cinem dichten Glianetzwerk.

An der Basis des Thalamus sind die basalen Opticusganglien gut erhalten, nur dic Subst. molec. ist etwas reduziert. Auch die Gansersche Kreu$z$ ung hat nicht gelitten.

Stärker degeneriert ist wieder das Corp. ma mmillare. Die Ganglienzellen seines lateralen Kernes sind meist zugrunde gegangen und auch im medialen ist dic Zahl vermindert und viele sind regressiv verändert. Der Körper ist auf ein Drittel seines Volumens zurückgegrangen. Die Fornixfaserung fehlt völlige, das Vieqd'Azyrsche Bündel ist stark reduziert; über das Haubenbündel vermag ich kejne Angaben zu machen.

Das zentrale HöhJengrau zeigt im oralen Teil des 3. Ventrikel keine Degeneration, im kaudalen ist es ärmer an Canglienzellen wie an der entsprechen. den Stelle der linken Seite.

Von den Nervenfasern des Thalamus ist der Stabkranz in allen seinen 'T'eilen völlig zugrundegegangen; dementsprechend sind auch die Einstrahlungen des Stabkranzes in die Thalamuskerne, das Stratum zonale und zum größten Teil die Lam. med. ext. zugrunde gegangen. Auch die Lam. med. int. ist bis auf geringe Reste geschwunden. Die Nervenfasern, die in den Kernen des Thalamus noch vorhanden sind, kommen sämtlich vom Linsenkern (s. oben) und von der Haube her, wo es hauptsächlich die mediale Schleife und das Mark des roten Kernes ist, dir dem Thalamus Nervenfasern zuführen. Die Mehrzahl der Fasern der medialen Schleife endet im $X$. vent. $c$ und bildet hier, untermischt mit Fasern des lateralen Markes des roten Kerns das dichte Fasergeflecht, das sich in diesem Kern findet. Fin kleiner Teil der Schleifenfasern bildet mit Fasem aus dem Linsenkern den vorhandenen Rest der Lam. med. ext. und zieht in die anderen ventralen Thalamuskerne (vent. ant.); ob Fasern der Schleife auch zum medialen Kern gelange'n, ist an meinen Präparaten nicht zu unterscheiden, zwischen $X$. vent c und med. b sind relativ reichliche Faserverbindungen durch die Lam. med. int. vorhanden. Im caudalen $A$ bschnitte des Thalamus kommt es an cireumseripter Stelle in der Frontalebene des oralen Pols des ('orp. genic. lat. nochmals zur Bildung einer Lam. med. ext. durch Faserbündel aus dem (orp. genic. lat., welehe zum Pulvinar ziehen. Die Fasem des dorsolateralen Marks des roten Kernes enden hauptsächlich im $\mathrm{N}$. med. $\mathrm{e}$ und $\mathrm{b}$ und im Ventr. a unter Bildung einer dünnen Lam. med. int., von der feine Fasern bis zum Gangl. haben. gelangen (Fig. 5, 6). Das frontale Mark, soweit es erhalten ist, splittert seine Fasern auf in den frontal gelegenen ventralen Kerngruppen besonders den $N$. vent. ant.; cinige Faserbündel gelangen auch in den ventralen Teil des medialen Kerns, in dessen frontalen Abschnitt.

hehr stark degeneriert ist ferner das (Cor p. genic. lat. (Fig. 6). Im Spornund Hilusteil sind die nervösen Elemente größtenteils geschwunden. Von den Marklamellen sind im ventralen Teil noch einige Nervenfasern erhalten; an der basalen Seite findet sich eine schmale Schicht kleiner Ganglienzellen, sonst sind diese völlig geschwunden. Etwas besser erhalten ist der (iitterteil; die einstrahlenden Opticusbündel sind zwar erheblich verschmälert, aber doch erhalten. Die Ganglienzellen zeigen beträchtlichen Ausfall, immerhin sind noch einige größere Elemente und zahlreiche kleinere, besonders auch in den dorsalen Sichichten, vorhanden. Die Sehstrahlungen zur Hemisphäre sind völlig untergegangen. Aus dem dorsofrontalen Pol treten relativ zahlreiche Faserbündel und zichen grobenteils durch dis Lam. med. ext. dorsalwärts zum Pnlvinar (s. oben); 
ein Teil dieser Fasern aber biegt nach medial um, tritt mit den caudalen Fasern der Linsenkernschlinge in Verbindung und wendet sich zum dorsalen Mark des Nucl. subthal.

Der Tractus optieus hat um über die Hälfte an Volumen verloren (Fig. 4), und zwar ist nicht nur das Kaliber der Fasern, sondern auch die Zahl der Fasern geringer. Die Meynertsche Commissur ist gut erhalten.

Ebenso wie das laterale ist auch das mediale ('orp. genic. stark degeneriert. Die (ianglienzellen sind im oralen 'Teil bis auf einige kleine Zellen geschwunden, im caudalen sind etwas mohr erhalten, die aber regressive Veränderungen

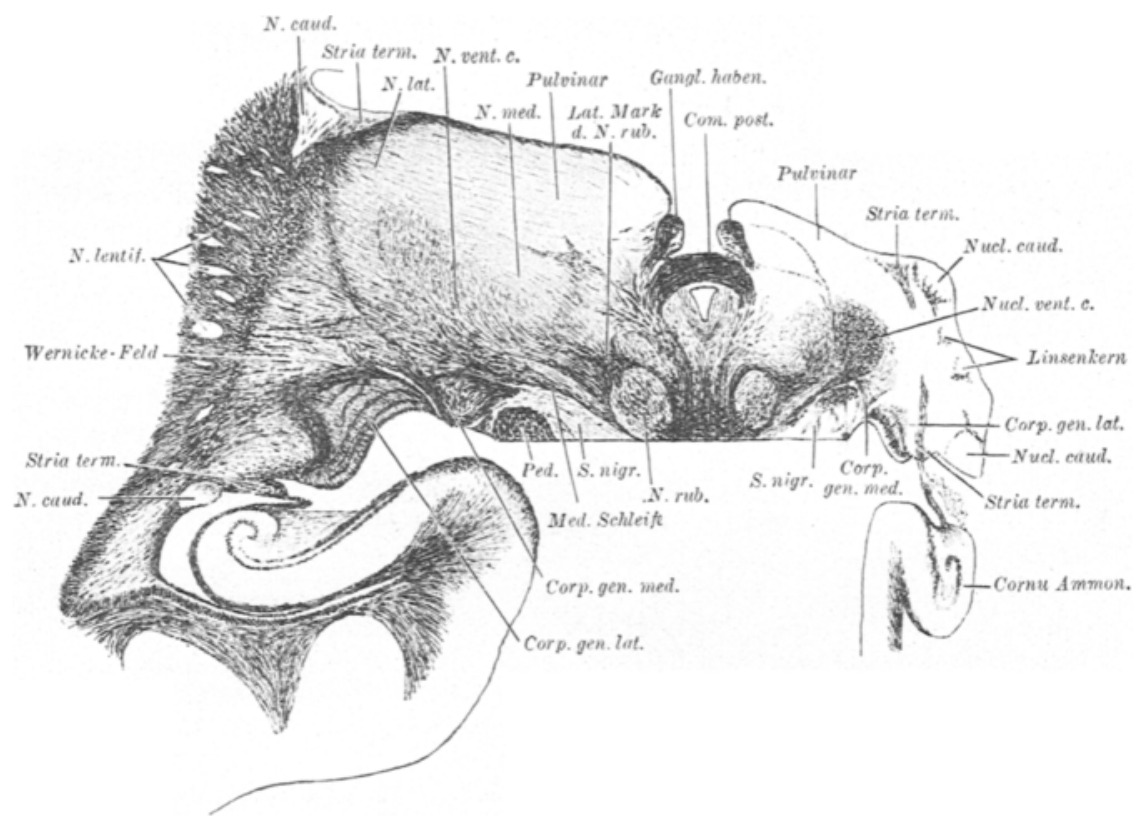

Fig. 13. Frontalsohnitt durch die zentralen ianglien, caudales prittel des Nucleus ruber.

Weigert-Pal. Vergr. $1: \stackrel{\bullet}{ }$.

zeigen. Die Strahlung zum T'(-mporallappen fehlt wieder völlig, wie überhaupt das ganze Wernickesche Feld gänzlich frei von Nervenfasem ist. Der Arm des

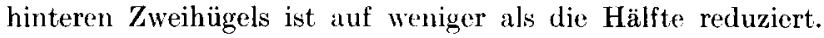

In der Regiosubthalamica hat die Zona incerta an Fasern wie an Zellen erhebliche Einbuße erlitten, weist aber noch eine Anzahl einfach atrophischer (ianglienzellen auf. Der Nucl. subthal. ist relativ gut erhalten (Fig. 5, 6). Nur die Molekularsubstanz ist verringert, so daß Zellen und Fasern, die beide kaum geschädigt sind, dichter zusammenliegen.

Der $\mathrm{N}$ ucleus $\mathrm{r}$ uber ist in seiner ganzen Ausdehnung an Volumen verringert (Fig. 5, 6), der frontodorsale Teil mehr wie der ventrocaudale. Die Verkleinerung wird hervorgerufen eimmal durch die Rarefizierung der Subst. molec., die am frontalen Pol und in den frontalen dorsolateralen Partien am stärksten ist, sodann durch eine Verschmälerung der Nervenfaserbälkchen, dir an den gleichen stellen wie auch im Zentrum des Kerns beträchtlich, aber auch in den ïbrigen Partien deutlich ist. Die Ganglienzellen sind im frontalen Pol, in den dorsomedialen und dorsolateralen Partien, frontal mchr wie caudal, sowie im Zentrum des Korns an 
Zahl etwas verringert, die erhalten gebliebenen vielfach atrophisch; im ventralen und ventromedialen Teil dagegen sind sie kaum verändert. Vom Mark des roten Kerns ist am stärksten reduziert das frontale, weniger das dorsolaterale, am geringsten aber immer noch deutlich, das mediale.

\section{Linke Hemisphäre.}

In ihr ist infolge der totalen Degeneration des Balkens das Centr. semiovale stark gelichtet. Der Ausfall des Balkens macht sich auch in der (iegend seiner Ausstrahlungen bemerkbar; so ist das Tapetum am Unterhorn auf etwa die Hälfte scines Umfangs zurückgegangen. Außerdem ist die Commissura ant. partiell degeneriert, besonders in ihrem temporalen 'J'cil, in wolchem sie weit über die Hälfte ihrer Fasern verloren hat. In übrigen sind in der linken Hemisphäre keine Degenerationen zu bemerken; die Z(llpräparate der Rinde waren leider verblasst.

\section{Vierhügelgegend.}

Das Corp. quadrigem. a nt. ist rechts auf die Hälfte scines Volumens \%11sammengeschrumpft. Im Stratum zonale sind keine, in Stratum optic. nur ver-

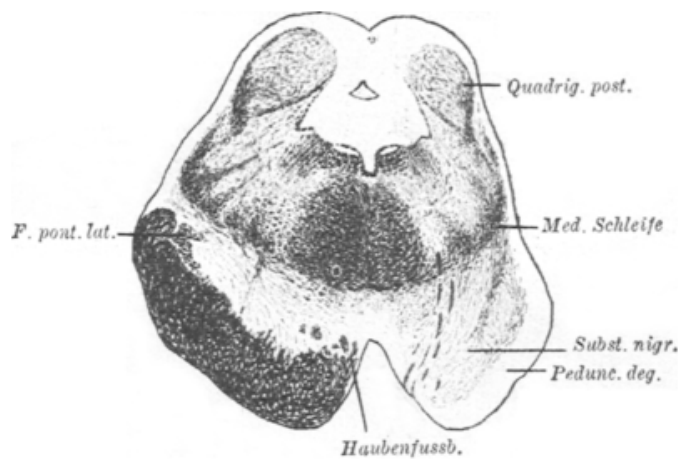

Fig. 7. Qnersehnitt, dureh die fiegend der hinteren Vierhïgel. Wejgert-Pal. Vergr. $1: 2 \underline{\text {. }}$

einzelt Fasern anzutreffon, auch dias Stratum lemnise. zeigt otwas Winbuße an Fasern. Die Molekularsubstanz ist in den oberflächlichen Sichichten beträchtlich reduziert. Von den (ianglienzellen sind die großen in den oberflächlichen fichichten an Zahl vermindert. Das rechte tiefe Mark steht dem linken nur wenig nach.

Das Corp. quadrigem. post. ist rechts ebenfalls kleiner wie links. 1)ie Nervenfasern sind in allen ihren Anteilen reduziert, am meisten die zum Brachium ad corp. gen. med., aber auch die Eigenfasem sind in großer Zahl ausgefallen. Die Ganglienzellen haben hauptsächlich in den dorsalen Teilen gelitten; ihre Anzahl ist hier verringert, sie sind vielfach atrophisch und auch sklerotisch verändert. Die Ganglienzellen in den ventralen Partien sind ziemlich gut erhalten.

Das zentrale Höhlengra u nimmt rechts ein gegen Jinks nur wenig kleineres Areal ein. Die Ganglienzellen zeigen rechts keine Veränderungen, nur die Subst. molec. ist in geringem (irade reduziert. Der Nucl. dors. tegm. ist gut erhalten.

Die Zellen des Nucl. Darkschewitsch und oeulomot. sind frei von dege. nerativen Veränderangen. Etwas reduziert ist die rechte Iaterale Haubengegend. Die Nervenfaserbündel sind dünner und liegen dichter beicinander. Das prädorsale Bündel ist rechts, nach der Kreuzung links, etwas ärmer an Fasern. Das zentrale $\mathrm{H}$ a ubenbündel zeigt rechts dünnere Faserbündel wie links. Das Gangl. mesence ph. lat. hat rechts eine geringe Einbuße an Zellen crlitten. Das dorsale Längsbiindel ist nicht geschädigt. 
Die mediale Schleife ist rechts in mäBigem Grude atrophisch und hat augenseheinlich auch einen geringen Ausfall von Nervenfasern. Die Gegend des tektospinalen Bündels ist rechts kaum ärmer an Fasern wie links. Der Bindearm ist nach dor Kreuzung links auf die Hälfte seines Volumens reduziert.

In Fußteil der Vierhügelgegend ist der rechte Pedunculus gänzlich ausurefallen. Seinen dorsalen Teil durchziehen in mannigfachen Windungen Fasern, die der Substantia nigra zugehören.

Die Śubstantia nigra ist rechts in ihrem oralen Teile mehr geschädigt wie im caudalen. In jenem ist "in großer Teil ihrer Ganglienzellen geschwunden, in diesem ist der Zellausfall nur gering. Die erhaltenen Zellen sind allermeist einfach atrophisch. Die Molekularsubstanz ist besonders in dem oralen Teil stark reduziert. In caudalen Teil sind zahlreiche Nervenfaserzüge vorhanden, die mannigfach gewunden sind und teils von der Linsenkernschlinge, teils von der Haube durch die mediale Schleife hindurc heinstrahlen; caudalwärts stehen sie mit den dorsalen Querfasern der Brüeke in Zusammenhang.

Brücke.

Die $\mathrm{H}$ a ubenregion der Brücke ist rechts an Unfang etwas geringer wie links. Die Reduktion ist bedingt durch eine geringe Atrophie der zentralen Haubenbahn, der Substantia retic. der medialen und lateralen Schleife. Auch das Sch ïtzesche Bündel ist rechts fisserärmer wie links.

Von den Kermen der Haube sind die Hirnnervenkerne gut erhalten; nur im Nucl. facialis ist links die Subst. molec. etwas reduziert und seine Zellen sind leicht atrophisch. Der Korn der lateralen Schleife ist rechts kleiner wie links; seine Zellen sind an Zahl etwas verringert und atrophisch; es finden sich auch sklerotische Zellen. Auch die obere Olive ist rechts in geringem Grade atrophisch. Der $\mathrm{Nucl}$. retic. tegm. weist links weniger Zellen auf wie rechts, die orhaltenen sind einfach atrophisch; die gleiche Veränderung ist am linken $\mathrm{Nucl}$. trapezoid. vorhanden. Das retikuläre Grau der Nchleifenschicht hat rechts eine sehr bedeutende Einbuße erlitten, es ist auf mehr als die Hälfte seines Umfangs zusammengeschrumpft und hat die entsprechende Anzahl Ganglienzellen verloren; die erhaltenen Ganglienzellen weisen vielfach sklerotische Veränderungen auf.

Die FuBetage der Brücke hat rechts beträchtlich an Volumen verloren durch den gänzlichen Ausfall der Pyramide; dieser hat auch Lücken in dem medialen Teil der medialen Schleife im frontalen Brückenabschnitt zur Folge.

Die Querfasern der Brücke sind im dorsalen Teil der Fußetage auf den beiden seiten wenig different, im mittleren und ventralen 'Teil aber rechts erheblich zahlreicher wie links. Die a ufsteigenden Raphefasern sind im ganzen spärlicher wie in normalen Präparaten; sie wenden sich in der Haube nach links zahlreicher als nach rechts. Der Bröickenarm ist links etwa auf die Hälfte des rechten reduziert.

Die feinen Nervenfasergeflechte und die Molekularsubstanz haben in der rechten Hälfte der Fußetage eine sehr beträchtliche Einbuße erlitten. An besten, wenn auch nicht in normaler Stärke, sind sie erhalten in den medialen Partien in der Nachbarsehaft der Raphe, fast völlig untergegangen sind sie in den Zonen zwischen den Zügen der Pyramide und im dorsolateralen Teil des frontalen Brückendrittels. Erheblich reduziert sind sie im dorsolateralen Teil der caudalen zwei Drittel des Brückenfulses und im lateralen 'Teil, etwas bessel' erhalten, aber auch noch crheblich reduziert, im dorsalen und ventralen Abschnitt; der laterale Abschnitt ist frontal, der dorsale caudal etwas besser erhalten. Ent- 
sprechend der Stärke des Ausfalls der Faseraufsplitterung ist dio Stärke der Schädigung der Ganglienzellen des BrückenfuBes. Nirgends ist eine Massondegeneration aufgetreten; überall aber sind dic Zellen gesehädigt, von einfacher Atrophie bis za schwerster Nklerose mit Ausfall zahlreicher Zellen. Mäßig atrophiseh sind die medialen Zellgruppen, stärker atrophisch die ventralen und ventrolateralen, atrophisch und teilweise sklerotisch die ventromedialen; zahlreiche sklerotische neben atrophischen Zcllen finden sich in den doromedialen, dorsalen (interstriären) und lateralen Partien; in den dorsolateralen des oralen Triks der

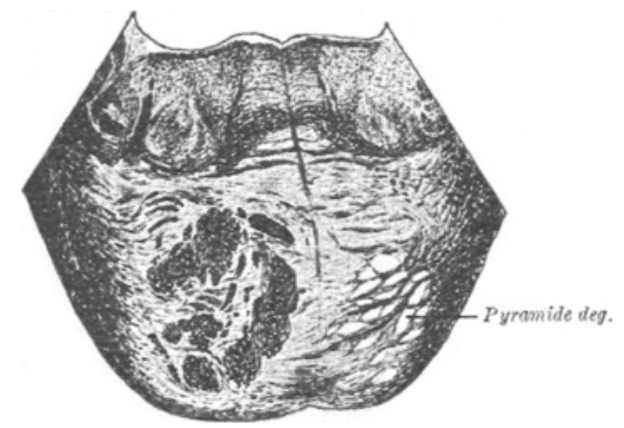

Fig. 7. Querschnitt durch die Brücke in Höhe der oberen Olive. Weigert-Pal. Vergr. $1: 2$.

Brücke und in den interpedunkulären Sehiehten ist die Zahl der Zellen erheblicher vermindert und dic Mehrahl der erhaltenen hochgradig skleresicrt.

\section{Kleinhirn.}

Die linke Kleinhirnhemisphäre ist um etwa ein Drittel kleiner als die rechte; die Verkleinerung betrifft hauptsächlich den Nucl. dentatus und die Hemisphäre, weniger den Wurm. Der Nucl. dentatus ist auf etwa die Hälfte seincs Volumens reduziert; dic Sichleifen seines Bandes sind verschmälert und dicht aneinander gelagert. Vließ und Marklager sind stark gelichtet. Die Ganglienzellen sind an Zahl etwas geringer, fast sämtlich atrophisch und zum 'Teil auch sklerosiert. Das Mark der Hemisphäre ist weniger reduziert wio das des Nucl. dentatus; die Markstrahlen der Läppchen sind sogar breiter wie in der rechten Hemisphäre, dafür aber erheblich kürzer, ähnlich wie bei der familiären Kleinhirnataxie. Stark gelichtet ist überall die Körnerschicht und auch die Purkinjeschen Zellen sind spärlicher und zu einem großen 'Teil der Atrophie und stärkeren regressiven Veränderungen verfallen. Dic Molekularschicht ist etwas versehmälert (Re. duktion der Molekularsubstanz).

Diese Veränderungen sind in den dorsalen Lappen der Hemisphäre etwas stärker wie in den ventralen, in den Läppehen der Peripherie wieder stärker wie in der zentralen; aich im Flocenlus sind sie beträchtlich. Im linken Teil des Ober- und Unterwurms sind sie viel weniger entwickelt und erstrecken sich hier auch auf den rechten Wurm in goringem Maße (Verschmälerung der Körnerschicht, teilweise Atrophic der Purkinjeschen Z(llen).

\section{Modulla oblongata.}

Total ausgefallen ist hier wiederum die Pyramide; durch ihr Gebict zichen zahlreiche Faserbündel von der Fibr. areif. ext. her zam Vließ der Olive und zur Ruphe. Erheblicher reduziert sind sodam die Kleinhirnanteile der Medulla: Das Corp. restif., die Fibr. cerebello-oliv., die innere Abteilung des Klein- 
hirnstiels und auch die Kleinhirnseitenstrangbahn zeigen links Atrophie und Faserausfall. Dia rechte untere Olive ist geschrumpft; ihr Vließ ist weniger gelichtet wie ihr Mark. Ihre Ganglienzellen sind meist atrophisch und teilweise sklerosiert. Einzelne sklerotische Zellen trifft man auch in der linken Olive an. In den Py ramidenkernen ist rechts dio Substant. molec. reduziert und die Ganglienzellen sind einfach atrophisch. Die Fibr. arcif. ext. haben rechts ebenfalls Faserausfall erlitten.

Starken Faserausfall zeigt von der Brücke bis zum caudalen Ende der unteren Olive die rechte zentrale Haubenbahn; etwas reduziert ist auch dic rechte Subst. retic. In der rechten Subst. gelat. ist die Molekularsubstanz verringert. Die Ganglienzellen des Seitenstrangkernes sind links meist atrophisch.

Das Areal der Schleifenschicht ist rechts um etwa ein Viertel seines Umfangs verringert, nicht nur dirch Kaliberreduktion, sondern auch durch Ausfall

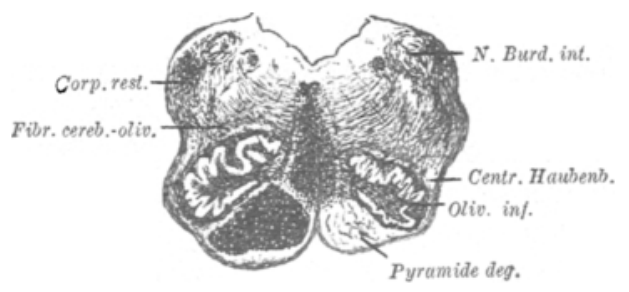

Fig. 9. Querschnitt durch die Medulla obl. in Höhe des Hypoglossuskerns, Weigert-Pal. Vergr. $1: 2$.

von Fasern. In dem Gollschen und inneren Burdachschen Kern der linken Seite findet sich eine Atrophic und teilweise sklerose der Ganglienzellen, während der äuBere Burdachsehe Kern nur eine geringe Atrophie seiner Zellen aufweist.

Die übrigen Kerne der Medulla obl., insbesondere die der Hirnnerven, sind nicht verändert.

Fassen wir die Krankengeschichte kurz zusammen: Eine bis dahin hinsichtlich des Nervensystems gesunde Frau macht in ihrem 25.-29. Lebensjahre eine Serie von epileptiformen Anfällen durch, der eine Schwäche in den Extremitäten der linken Seite und eine Hemianästhesie auf der gleichen Seite vorausging; nach ihrem Abklingen war eine residuäre Hemiplegie und eine Hemianästhesie der linken Seite vorhanden. Im 30. Lebensjahr trat eine 2. Seric epileptiformer Anfälle in Form eines Status epilepticus auf, die 2 Wochen dauerte und eine das ganze Leben anhaltende bilaterale linksseitige Hemianopsie zur Folge hatte, und im 32. Lebensjahr eine dritte Serie in der Dauer von 1 Monat wieder in Form des Status, die weitere dauernde Ausfälle von seiten des Nervensystems nicht hinterließ, nach der aber für mehrere Wochen konjugierte Deviation der Bulbi nach rechts mit beiderseitiger Ptosis, starke Facialisparese und völlige Lähmung der Extremitäten der linken Seite bestand. Abgesehen von einer kurzen Attacke leichter epileptiformer Anfälle im 34. Lebensjahr blieb dann die Frau bis zu ihrem Tode, der im 40. Lebensjahr eintrat, frei von akuten Störungen von seiten 
des Nervensystems. Bei der Sektion fand sich das Mark der rechten Hemisphäre in ganzer Ausdehnung zerstört und an seiner Stelle eine zum Teil mit trüber Flüssigkeit gefüllte Cyste.

Während der drei Anfallsserien muß das Hemisphärenmark zerstört worden sein. Was war zunächst der Ursache derZerstörung? Dem ganzen Verlauf nach kommt nur eine langsam wirkende Noxe in Betracht; da Tumor nach dem pathologischen Befund auszuschließen ist und das Mark durch Erweichung zugrunde gegangen sein muß, bleibt nur übrig ein inkompletter Verschluß der arteriellen Gefäße. Herzerkrankung und andere ursächliche Momente für Thrombenbildung waren nicht vorhanden, dagegen erkrankte die Frau während ihres Anstaltsaufenthalts an breitem Kondylom, so daß die naheliegendste Annahme die ist, daß gummöse Prozesse der Gefäßwände das Lumen der arteriellen Gefäße zum größten Teil verlegt haben. Dies würde auch erklären, daß bei der Sektion die Gefäße sämtlich gleichmäßig durchgängig gefunden wurden; die gummösen Prozesse waren zur Abheilung gekommen. Der Umstand, daß die Gefäßlumina nicht vollständig verschlossen wurden, erklärt einmal das Auftreten so zahlreicher epileptiformer Anfälle in jeder Erweichungsattacke und ferner das Erhaltenbleiben der Rinde, die nur durch sekundäre Veränderungen geschädigt ist; zu ihrer Ernährung reicht bekanntlich eine geringere Spannung in den arteriellen Gefäßen aus, als sie das Mark nötig hat.

Aus dem Sektionsergebnis folgt, daß sämtliche Arterien der rechten Hemisphäre von gummösen Prozessen befallen gewesen sein müssen, und zwar in ihren Hauptstämmen, jedoch distal von den Ästen, die sie zum Zwischen- und Mittelhirn entsenden; diese Gegenden weisen nur sekundäre Veränderungen auf.

In welcher Reihenfolge die Arterien erkrankt sind, darüber geben die klinischen Folgeerscheinungen Aufschluß: während der 1. Attacke, die Hemiplegie und Hemianästhesie hinterließ, war es die Art. foss. Sylv., während der 2. die Art. cerebri post. (Hemianopsie) und während der 3. die Art. cerebri ant. Ob bei der l. Attacke der Stamm der Art. foss. Sylv. erkrankt war oder die Hauptäste nacheinander erkrankten, läßt sich bei der mangelhaften Anamnese nicht sagen. Bei der langen Dauer der Attacke und dem Fehlen einer völligen Lähmung der linken Seite am Anfang ist aber das letztere wahrscheinlich; der erste befallene Ast würde dann der 3. gewesen sein, da Hemianästhesie und Schwäche der linken Seite die ersten Symptome waren.

Was die Attacken im einzelnen betrifft, so gilt zunächst für alle, daß die Erweichung des Marks infolge des unvollständigen Verschlusses der Gefäße relativ langsam vor sich gegangen sein muß; der Lähmung durch Unterbrechung der Fasern ging eine ziemlich lange Zeit der 
Reizung voraus, die sich in zahlreichen epileptiformen Krämpfen äußerte; in der 2. Attacke wurlen bis 176 Anfälle an einem Tage gezählt.

Uber die 1. Attacke ist weiteres Bemerkenswertes als das bereits erwähnte nicht bekannt.

In der 2. Attacke (Erweichung des Markes des Occipitallappens aus der basalen Hälfte des Temporallappens) zeigten die epileptiformen Anfälle folgendes Bild: Im Anfang des Status traten linksseitige Parästhesien und Zuckungen auf, die in der linken Hand begannen und sich sukzessive auf den Arm, die linke Gesichtshälfte, in geringem Grade auch auf das linke Bein ausdehnten. Im weiteren Verlauf gingen sie dann unter anfänglicher Drehung des Kopfes und der Augen nach links in allgemeine Krämpfe über, die sich von gewöhnlichen epileptischen nur durch ihren linksseitigen Beginn unterschieden. In der 2. Hälfte des Status traten damm zunächst nur in Gesicht und Augen klonische Zuckungen auf, während die Extremitäten in tonischer Starre verharrten; am Schluß des Status zeigten sich weiter allgemeine tonisch-klonische Krämpfe von ganz unregelmäßigem Beginn und Verlauf.

Im Beginn des Status waren sonach ganz typische Jacksonsche Anfälle vorhanden, wie wir sie bei Reizung der Rinde zwischen mittlerem und unterem Drittel der vorderen Zentralwindung sehen. Später wurden die lokalisatorischen Zeichen undeutlicher, klonische Krämpfe aber waren während des ganzen Status auch in den linksseitigen Extremitäten vorhanden. Nun war aber von der der ersten Attacke eine Hemiplegie mit Facialisparese zurïckgeblieben, es ist also anzunehmen, daß während derselben das Mark der Zentralwindungen zerstört worden war, zumal die Äste der Art. foss. Sylv. noch über die vordere Zentralwindung hinaus zum Stirnhim sich erstrecken. Es hätten also nach den experimentellen Erfahrungen bei Tieren in der linken Seite keine klonischen Krämpfe, geschweige denn typisch Jacksonsche Anfälle mehr auftreten dürfen. Wie ist dieser Widerspruch zu erklären? Ich glaube, daß für die Erscheinung die Fibr. propriae der Rinde verantwortlich zu machen sind, die von der sekundären Degeneration verschont geblieben sind und eine Verbindung herstellten zwischen den Zentralwindungen und dem damals noch nicht zerstörten Stirnhirn; vom Stirnhirn aber war eine Reizübertragung durch Brücke, Kleinhirn usw. gegeben. Möglich wäre es auch, daß bei dem ersten Insult infolge von Kollatralen der Art. cerebr. ant. Reste der vorderen Zentralwindung erhalten waren. Daß ein Reizzustand im Occipital- und Temporallappen sich auf die Zentralwindungen iiberträgt, und zu Jackson schen Anfällen führt, ist ja nichts Ungewöhnliches.

Die Ausfallserscheinungen nach Ablauf der 2. Attacke bestanden in linksseitiger bilateraler Hemianopsie, die dauernd bestehen blieb, und in Blicklähmung des linken Auges nach links, oben und unten, 
Dysarthrie und starker Facialisparese links; die letztgenamnten Symptome schwanden im Laufe zweier Wochen. Auf Dysartherie und Facialisparese komme ich noch weiter unten zu sprechen. Sehr a uffällig ist die auf das linke Auge beschränkte Blicklähmung. Der Bulbus war in starker Konvergenzstellung fixiert. In Mittelhirn und Brücke wurden keine Veränderungen gefunden, die diese Erscheinung erklären könnten. Die klonischen Krämpfe während des Anfalls erstreckten sich auf beide Augen. 1ch kann bei alledem nur die Tatsache registrieren, daß es bei Ausschaltung des Occipitallappens zur Blicklähmung des gegenüberliegenden Bulbus kommen kann, ohne eine Erklärung dafür geben zu können; höchstens könnte man daran erinnern, daß du Bois-Reymond und Silex vom Facialisfeld aus bei Reizversuchen auch nur Bewegungen des Bulbus der anderen Seite erhielten (Neurol. Centralbl. 18999).

Die 3. Attacke - Zerstörung des Markes des Stirnhirns und der medialen Teile der Hemisphäre oberhalb des Balkens bis zum cuneus - bietet, wie zu erwarten, keine solche scharfen Lokalsymptome wie die ersten beiden. Die klonischen Krämpfe beschränkten sich auf die linke Gesichts- und Halsmuskulatur und die Augen, in den linksseitigen Extremitäten traten tonische Beuge- und Streckkrämpfe auf; nur beim Abklingen des Status kam es zu Zuckungen in Hand und Fuß der linken Seite.

Bemerkenswert scheint mir, dlaß hier wie besonders während der kurzen Serie leichter epileptiformer Anfälle im 34. Lebensjahr lokalisierte Klonismen im linken Arm und Bein auftraten. Zur Zeit der kurzen Serie war sicher der Zerfallsprozeß des Hemisphärenmarks abgeklungen, wenn auch aus irgendeiner Ursache ein Reizzustand im Gehirn bestand; die Anfälle konnten durch mechanische Einwirkung (Wenden des Kopfes) hervorgerufen werden. Wem man nicht annehmen will, daß mittels der Fibrae propriae von den Zentralwindungen aus durch Thalamus, roten Kern, Kleinhirn usf. der Reiz zu den tiefergelegenen Zentren gelangt ist, so wäre damit dargetan, da 3 es a uch oh ne Rinde $\mathrm{zu}$ Jacksonschen Anfällen kommen kann. Wie weit aber zur Zeit dieser kurzen Serie die Fibrae propriae der Zentralwindungen noch erhalten waren, läßt sich nicht mit Bestimmtheit sagen; bei der mikroskopischen Untersuchung funden sie sich zum größten Teil, wahrscheinlich aus mechanischer Ursache, zerstört.

Als Ausfallserscheinung blieben nach der 3. Attacke zurück: Konjugierte Deviation der Bulbi nach rechts mit Ptosis, die auf dem linken Auge stärker war wie auf dem rechten, Dysarthrie, starke Facialisparese und eine komplette Jähmung der linksseitigen Extremitäten. Alle diese Ausfallserseheinungen bildeten sich im Laufe zweier Monate zurïck. 
Die konjugierte Deviation und die Ptosis erklät sich unschwer durch den Ausfall der Rindenfelder für dic Augenbewegungen im Stirnhirn, wie sie die Reizversuche bei Affen ergaben. Auch beim Menschen ist diese Frscheinung bei apoplektischen Herden des Stimhirns wiederholt beobachtet worden (vgl. Murri, Orig. della deviazione etc. Riv. crit. d. clin. med. Firenza I).

Die völlige Lähmmg ater Extremitäten ist wohl als Diaschisiserscheinung aufzufassen, die subcorticalen Zentren wurden ihrer letzten Rindenverbindung mit der Zerstörung des Stirnhirnmarks beraubt; Arm und Bein verfielen so der schlaffen Lähmung, wie initial bei jeder Hemiplegie, bis die Neuorientierung vollzogen war.

Nich der 2. und 3. Attacke trat starke linkssoitige Facialisparese und Dysarthrie auf; wir kömnen wohl mit Sicherheit annehmen, dal 3 dies auch nach der I. der Fall war, denn mäßige Facialisparese wurde schon mehrere Monate nach dem Abklingen der 1. Attacke gefunden. Wahrschẹinlich hat auch während oder nach der 1. Attacke Deviation der Bulbi bestanden. Fïr diese ist ihr jedesmaliges Auftreten leicht zu erklären, da im Gyr. angul., im Occipital- und Frontallappen Zentren für die Augenbewegungen experimentell und klinisch festgestollt sind. Anders ist es mit dem Facialis, dessen Lähmung wohl die Dysarthrie mit veranlaßte. Fiir ihn ist nur das Kentrum am Ful.3 der vorderen Zentralwindung bekannt und dieses wurde höchstwabrscheinlich schon in der 1. Attacke von den subcorticalen Zentren abgesehnitten; wäre dies aber auch erst in der 3 . Attacke vollständig geschehen, so traten doch in der 4. Attacke der leichten epileptiformen Anfälle die stärksten Zuckungen im linken Facialisgebiet auf, wo sie auch in der 3. mit am stärksten gewesen waren. Wine Erklärung für die jerlesmalige Verstärkung der Facialisparese ist schwer \%u geben, wenn man nicht annehmen will, dab der Reiz durch die Fibrae propriac zum Stimhirn und von da zum Pons geleitet worden ist, so clak wir nach der 2. Attacke eine Erschöpfung, nach der 3. cine Diaschisisparese vor uns hätten. Zur Wrklärung der Zuckungen während der 4. Attacke würle man beim Facialis mit mehr Recht auf die Wibrae propriae zurickgreifen können wie beim Arm und Bein, da die Fibrae im Facialisgebiet noch nach dem Tode in gröberer Menge vorhanken waren. (Ob) aber auf diesem Wege regelrechte Jacksonsche Aufälle ausgelöst werclen kömnen, ersoheint mir sehr zweifelhaft.

Wenn wir nun zur Besprechung der Residuärerscheinungen nach Ausfall der rochton $\mathrm{Hem}$ is phäre übergehen, so ist zunächst die Frage zu beantworten, die wir schon gestreift haben, inwieweit Impulse von der Rinde her zu tieferen Hirnteilen gelangen konnten. Die Rinde des Gyr. rect, der Area olfact. und des oralen Teiles des Gyr. hypoeamp. steht mit dem Zwischenhim vom frontalen Pol des Nule. 
caud. bis zum caudalen Pol des Nucl. amygd. in Verbindung. Aus diesen Rindenteilen treten einige Fasern zur Subst. perforat. ant., zum unteren Thalamusstiel und zum Nucl. anygd.; aus dem Ammonshorn ziehen einzelne Fasern zur Fimbria. Damit ist der Connex zwischen Rinde und tiefen Hirnteilen erschöpft. An der dorsalen und lateralen Peripherie der rechten Hemisphäre ist außerdem die Rinde so schwer geschädigt, daß von der Fibrae propriae wie von den Rindenschichten wenig oder so gut wie nichts mehr übrig geblieben ist. Wenn daher ein Einfluß der Rinde auf tiefer gelegene Hirnteile überhaupt stattgefunden hat, so muß er ein sehr geringer gewesen sein. Praktisch dürfte er jedenfalls für die Residuärerscheinungen nicht in Betracht kommen.

Als Residuärerscheinungen wurden beobachtet:

1. Eine linksseitige Hemiplegie, die sich von der bekannten Form in nichts unterschied, mit mäßiger Facialisparese links und Steigerung der Sehnenreflexe. Die Sprache war etwas unbeholfen und explosiv, die Augenbewegungen waren nach allen Richtungen frei.

2. Eine linksseitige He mianästhesie, die an den distalen Teilen der Extremitäten am stärksten ausgebildet war. Sie bestand in einer Abstumpfung der Berührungs- und Temperaturempfindung; die anfängliche Analgesie wandelte sich allmählich in eine Hyperalgesie. Drucksimn und Lokalisation waren nur wenig, stark die Tiefensensibilität gestört.

3. Eine linksseitige bilaterale Hemianopsie, die auf dem linken Auge einen größeren Umfang hatte, wie auf dem rechten.

Ob eine Störung des Geruchs oder Geschmacks auf der linken Seite vorhanden war, wurde versäumt zu prüfen.

Anatomische Folgender Zerstörung des Hemisphärenmarks.

In der Hirnrinde finden wir sekundäre Veränderungen von sehr verschiedener Stärke. Sie ist in der dorsalen Hälfte der Hemisphäre viel stärker geschädigt als in der ventralen, die Scheitel der Gyri wieder mehr wie die Furchenteile; in den mittleren Zweidritteln der dorsolateralen Peripherie geht die Schädigung im Scheitel der Gyri meist so weit, daß von nervösen Bestandteilen nur noch kümmerliche Reste oder gar nichts mehr vorhanden ist. Diese Schädigung kann nicht die Folge des Markausfalls sein, sie ist jedenfalls durch mechanische Ursachen (Dehnung), vielleicht auch durch meningitische Veränderungen hervorgerufen worden; die Pia fand sich bei der Sektion an diesen Stellen erheblich verdickt. Fïr die Beantwortung der Frage, welche sekundären Veränderungen die Zerstörung des Hemisphärenmarks in der Rinde hervorruft, kommen nur die besterhaltenen Rindenstellen in Betracht. In diesen finden wir, daß die Schicht der g ro ße n P y ra mide nzellen, in den Zentralwindungen auch die Riesenpyramiden, 
gänzlich ausgefallen sind; die Schicht der Körner-und polymorphen Zellschicht ist statt geschrumpft durch hochgradige Reduktion der Molekularsubstanz und Atrophie, Sklerose und partiellen Schwund der Ganglienzellen; die Molekularschicht und die der kleinen Pyramiden weist keine erheblichen Veränderungen auf. An der Innenfläche der Rinde findet sich eine Schicht von Nervenfasern, die Fibrae propriae, die eine Dicke bis zu fast $1 \mathrm{~mm}$ erreicht; ihre Fasern strahlen in die stark reduzierten radiären Markstrahlen der Rinde ein; die Baillargerschen Streifen sind noch deutlich erkennbar aber ebenfalls beträehtlich reduziert.

Die mit dem Untergang des Hemisphärenmarks unterbrochenen Commissuren, Stabkranz- und Projektionsfasern sind auch in ihrem Verlauf außerhalb des Hemisphärenmarks gänzlich zugrunde gegangen. Dies hat einmal zur völligen Degeneration des Balkens geführt, dessen Ausfall eine sehr deutliche Rarefizierung des Marklagers der linken Hemisphäre verursacht hat. Sodann ist die in nere Kapsel bis a uf die Linsenkernfaserung gänzlich zugrunde gegangen und mit ihr der Pedunculus und die Pyramidenbahn; die gut erhaltene Linsenkernfaserung setzt sich zusammen aus Fasern, die vom Linsenkern zum Nucl. caudat. und solchen, die vom Linsenkern zum Thalamus und zur subthalamischen Region ziehen, und findet sich in wechselnder Stärke in der ganzen Ausdehnung des oralen Teils der inneren Kapsel (lenticulo-striärer Teil, Knie und lenticulo-optischer Teil). Die Verbindung zwischen Linsenkern und Thalamus beschränkt sich also nicht auf die Fasern, die man gewöhnlich unter dem Namen Linsenkernschlinge zusammenfaßt, sondern ist eine viel ausgedehntere. Partiell degeneriert ist die Commissura ant.; ihr temporaler Anteil ist völlig geschwunden.

Völlig zugrunde gegangen ist ferner der rechte Fornix und mit ihm der Alveus des Ammonshorns, wohl eine Folge der Erweichung des Markes des Gyr. fornic. und der hochgradigen Degeneration des Ammonshorns.

Der Untergang des Stabkranzes hat dureh retrograde Degeneration zum völligen Schwund der Ursprungszellen seiner Fasern geführt, wodurch sich die schweren Veränderungen im Thalamus und in den beiden Kniehöckern erklären. Von den Thalamuskernen sind die am schwersten geschädigten der laterale Kern, das Pulvinar, der hintere Kern und der dorsale Abschnitt des oralen Teiles des medialen Kerns, in denen nur noch äußerst wenig von nervöser Substanz erhalten ist; in 2. Reihe kommen der vordere Kern und die fronto-lateralen Teile des ventralen Kerns (vent. ant., a, c); am besten erhalten sind der mediale Abschnitt 
des ventralen Kerns (vent. b) und der caudale Teil des medialen (med. b, c). Im Corp. mammillare ist der laterale Kern so gut wie völlig, der mediale teilweise degeneriert. Nicht geschädigt ist im Thalamusgebiet nur die Stria terminalis, die Taenja tha la mi und das Gangl. habenulae, wenig geschädigt die basalen Opticusganglien und die Gansersche Kreuzung.

Von den Kniehöckern ist das Corp. genic. lat. in seinem Hilus. und Spornteil fast völlig degeneriert, während der Tractusteil nur stark atrophisch ist. Das ('orp. gonic. med. ist hochgradig geschrumpft und größtenteils degeneriert.

Da, wo die untergegangenen Projektionsfasern enden, ist es vielfach zur Degeneration rler molekulären Substanz gekommen. Eigentümlicherweise ist das nicht der Fall an den motorischen Kernen der Hirnnerven (das Riickemmark wurde nicht untersucht), nur im linken lacialiskem fand sich eine Amleutung lavon. Hochgradig ist dagegen die Reduktion der Molekularsubstanz in der Fußetage der Brücke und auch im frontalen Pol des roten Kerns. Diese Redaktion hat wieder eine starke Sklerose und partiellen Schwund der Ganglienzellen des anschließenden Neurons zur Folge gehabt.

In der Brücke ist diese Veränderung am stärksten anzutreffen in den intraperlunkulären Abschnitten des Brïckengraus sowie in dem dorsolateralen des oralen Brückendrittels, ebenfalls hochgradig in den dorsomedialen und ventromedialen Abschnitten; gering ist die Reduktion in den medialen, ventralen und ventrolateralen Abschnitten, während sie in der lateralen und intrastrï̈ren noch einen ziemlich hohen Grad erreicht. Der Befund stimmt ziemlich gut mit dem von Borow icc ki nach experimenteller Pedunculusclurchtrennung erhobenen überein (vgl. anat. u. exp. Unters. über d. Brïckengrau usw. v. Monakows Arbeiten, Bd. II).

Noch ein Befund ist hier zu erwähnen, über dessen Entstehung ich mich einer Meinungsäußerung enthalte: das retikuläre Grau der Schleifenschicht der Brücke war in ähnlicher Weise degeneriert wie das Brïckengrau; es war stark zusammengeschrumpft, seine Zellen waren auf die Hälfte etwa reduziert und stark regressiv verändert. Man hat den Eindruck, als handle es sich auch bei diesem Grau um einen direkten Großhirnanteil.

Bei den weiteren sekundären Veränderungen ist die Genese nicht so eindeutig wie bei den aufgeführten. Ein großer Teil dieser Veränderungen besteht in einer einfachen Kaliberreduktion von Fasern und Zellen, die man gewöhnlich der Inaktivität zuschreibt; andrerseits sind aber auch stärkere regressive Prozesse vorhanden bis zum Schwund einzelner Zellen und Fasern. Wie weit dabei etwa retrograde Degeneration oder Vorgänge wie im Brückengrau oder andere dabei in Frage kommen, 
lasse ich dahingestellt. Für den verschicdenen Ciad der regressiven Veränderungen erscheint mir auch die Erklärung diskutabel, daß es je nach der Menge der aus dem zentralen Neuron zu den nächstfolgenden Neuronen ïbertretenden Fibrillen zur Atrophie, Sklerose oder Schwund in den Ganglienzellen der nächstfolgenden Neurone kommt.

Eine ziemlich erhebliche Atrophie zeigt der Streifenhügel und zwar der Nucl. caud. und das Putamen mehr wie der Glob. pallid. Das Vorkommen dieser Atrophie nach Hemisphärendefekt ist bezweifelt worden; sie wird aber auch schon von (ruveilhier nach alter ausgedehnter Atrophie einer Hemisphäre erwähnt (Anat. path. Tom. I, Liv. 5, 8). Nur sehr wenig verändert war hingegen der $\mathrm{Nucl}$. a mygd.

Ferner waren im Zwischenhim atrophisch die Zona incerta, die Subst. nigr., der Tract. opt., wenig das Corp. subthal.

In der rechten Hälfte des Mittelhirns erstreckte sich die Atrophie auf die Corp. quadrig. ant. und post. mit ihren Stielen, den Nucl. ruber, die zentrale Haubenbahn, die Subst. retic., das prädorsale Bündel (nach der Kreuzung links atrophisch), die mediale und laterale Schleife. In Pons und Medulla waren außer den beim Mittelhirn genannten Bahnen rechts atrophisch: das Schützesche Bündel, der Kern der lateralen Schleife, die obere Olive, die untereolive, die Pyramidenkerne, die Fibr. areiform. ext. und die Subst. gelatinosa der aufsteigenden Trigeminuswurzel; links der Binde- und Brückenarm, der Nucl. retic. tegm., der Nucl. trapezoid., das Corp. restif., die Kleinhirnolivenfasern, der Seitenstrangskern, der Gollsehe und dio innere Abteilung des Burdachschen Kerns.

Die Subst. nigr. weist in ihrem oralen Teil einen beträchtlichen Ausfall an Ganglienzellen auf, während or im caudalen gering ist und hier einfach atrophische Veränderungen vorherrschen. Im Nucl. ruber sind die regressiven Veränderungen am stärksten im frontodorsalen Teil, danach im Zentrum, die ventrocaudalen Teile sind wenig geschädigt. In beiden Vierhügeln sind am stärksten reduziert die dorsalen Teile; im vorderen Vierhügel ist vom Strat. opt. und zonale fast nichts mehr iibrig, in dem an diese Strata angrenzenden Grau ist die Molekularsubstanz stark geschrumpft und die Ganglienzellen zeigen Atrophie, Sklerose und partiellen Schwund. Von den Kernen der Hinterstränge ist der äußere Teil des Burdachschen Kerns, worauf auch v. Monakow bei seinen Untersuchungen über die sekundären Degenerationen nach Hemisphärendefekt aufmerksam macht, von der Atrophie fast völlig verschont geblieben. Endlich ist noch besonders hervorzuheben, daß die zentrale Haubenbahn von der Brücke abwärts viel stärkeren Faserausfall zeigte als in Brücke und Mittelhirn. 
$\mathrm{Zu}$ erwähnen sind noch die sekundären Veränderungen im Kleinhirn. Hier hat am stärksten gelitten der Nucl. dentatus und die Hemisphäre der linken Seite, während der Wurm weniger verändert ist. Marklager und Vließ des Nucl. dent. sind erheblich reduziert, etwas weniger das Hemisphärenmark. Im Band des Nucl. dent. findet sich Atrophie und Sklerose der Ganglienzellen. Die Rinde der Hemisphäre ist in allen 3 Schichten reduziert, sehr stark ist der Zellschwund in der Körnerschicht, weniger stark in der Purkinjeschen. Die Degeneration ist in den dorsalen Lappen stärker als in den ventralen, in den peripher gelegenen Läppchen stärker als in den zentralen. Von den Kleinhirnschenkeln ist am stärksten (auf etwa die Hälfte) reduziert der Bindearm, dann folgt in der Stärke der Atrophie der Brückenarm und endlich der zur Medulla obl. Der erhobene Befund stimmt gut überein mit einer Beobachtung von André - Thomas und Cornelius (Un cas d'atrophie crois. d. cervelet. Rev. neur. 1907), die diese Atrophie vorfanden bei einer ausgedehnten Schrumpfung der rechten Hemisphäre (besonders des Frontal-, Parietal- und Temporallappens). Sie halten die Atrophie für eine Kombination von Agenesie und Degeneration; für die Agenesie scheint mir wenig zu sprechen. Daß jedenfalls die halbseitige Kleinhirnatrophie eine reine sekundäre Veränderung nach Ausfall einer Hemisphäre sein kann, zeigt unser Fall.

Vergleichen wir unseren Befund mit experimentell gewonnenen Ergebnissen, insbesondere mit dem, was v. Monakow mehrere Monate nach der Operation bei dem Hunde fand, dem bald nach der Geburt die Hemisphäre mit Schonung der zentralen Ganglien abgetragen worden war (Exp. u. path. anat. Unters. über d. Haubenreg. usw. Arch. f. Psych. 27), so finden wir eine weitgehende Übereinstimmung der sekundären Veränderungen.

Die Differenzen seien hervorgehoben:

v. Monakow fand die vordere Commissur gut erhalten. Da der Schläfenlappen abgetragen war, ist dieser Befund nicht recht verständlich, wenn auch die basalen Teile des Stirnlappens teilweise erhalten waren.

Der rechte Fornix war nur atrophisch, nicht völlig geschwunden wie im vorliegenden Falle. Der Grund dafür ist jedenfalls darin zu suchen, daß die mediale Seite der Hemisphäre oberhalb des Balkens teilweise erhalten geblieben war, darunter der Gyr. fornic. Aus demselben Grunde war auch der Balken nicht völlig degeneriert.

Im Streifenhügel fand v. Monakow nur das mittlere Glied des Linsenkerns an Volum verringert. Aber auch in den anderen Teilen fanden sich regressive Veränderungen an Zellen und liasern, so daß es wohl auch hier zur Atrophie gekommen wäre, wenn das Tier längere Zeit am Leben erhalten worden wäre. 
Der Nucl. a mygd. war bei dem Hunde atrophisch; verursacht war wohl diese Atrophie durch den Wegfall der Strahlung aus der Hemisphäre vom Gyr. hypocampi her, die in meinem Falle erhalten war.

Die Degeneration der Thala muskerne zeigt nur wenig Verschiedenheiten, abgesehen ron dem Grade der Degeneration, der in meinem Falle ein höherer war. Nur bei den medialen Kernen differiert der Befund wesentlicher: $v$. Mon a kow fand med. c schwer degeneriert, med. b stark, med.. a wenig reduziert, während in meinem Falle der frontodorsale Teil des medialen Kerns viel mehr geschädigt war als der ventrocaudale.

Der Tract. opt. war beim Hunde gleichfalls atrophisch, Gangl. habenul., Taenia und Fase. retroflexus ebensowenig geschädigt. Über die Stria term. erwähnt $v$. Monakow nichts.

Im Mittel- und Nachhirn decken sich die Befunde ziemlich. Nur die Subst. nigr. fand ich im caudalen 'Teile besser erhalten, beim Hunde war sie in ganzer Auslehnung sehwer geschädigt.

Da Pedunculusteile aus dem Frontallappen noch erhalten waren, waren ferner beim Hunde das Brïckengrau und Kleinhirn weniger, die untere Olive gar nicht geschärligt.

Zum Schlusse hebe ich noch einiges Bemerkenswertes von den Befunden an den Nervenbahnen hervor:

Vom Streifen- zum Sehhügel ziehen, außer der Linsenkernschlinge, durch die innere Kapsel, soweit sic zwischen den beiden Kernen verläuft, wie oben schon erwähnt, Faserzüge, die im lenticulo-striären Teil der inneren Kapsel zu Bündcln verbunden, in den anderen Teil mehr gleichmäßig verteilt sind; sie treten aus dem Linsenkern an der dorsomedialen Fläche besonders am Scheitel und an den Marklamellen aus und sind bis in clen vorderen, lateralen, medialen und ventralen Thalamuskern zu verfolgen.

Der Nucl. amydg. sendet Fasern in die Gegend des unteren Thalamusstiels und in das ventrale Mark des Linsenkerns und steht jedenfalls so in derselben Weise mit dem Thalimus und den subthalamischen Gebieten in Verbindung wie der Streifenhügel.

Aus dem oralen Pol des Corp. genic. lat. treten gleichzeitig mit den Fasern zum Pulvinar auch solche zum dorsalen Mark des Corp. subthal.

Vom Sept. pelluc. zichen Fasern zum ('ingulum und zur Stria landisii, von welcher wieder Fasern ins (ingulum treten.

Uber die Endigung der medialen Schleife und des Marks des roten Kerns ist im mikroskopischen Teil schon das Nähere gesagt.

Herr Prof. v. Monakow hatte die große Giite, einen Teil meiner Präparate durchzusehen, mich Finsicht nehmen zu Jassen in einschlägige Präparate seiner Sammlung und mir mit Ratschlïgen beizustehen, wofür ich ihm auch an dieser Stelle meinen tiefgefiihltesten Dank ausspreche. 